\title{
Initial and final state temperatures of antiproton emission sources in high energy collisions
}

\author{
Qi Wang, Fu-Hu Liv* \\ Institute of Theoretical Physics and State Key Laboratory of Quantum Optics and Quantum Optics Devices, \\ Shanxi University, Taiyuan, Shanxi 030006, China
}

\begin{abstract}
The momentum or transverse momentum spectra of antiprotons produced at mid-rapidity in protonhelium $(p+\mathrm{He})$, gold-gold $(\mathrm{Au}+\mathrm{Au})$, deuton-gold $(d+\mathrm{Au})$, and lead-lead $(\mathrm{Pb}+\mathrm{Pb})$ collisions over an energy range from a few $\mathrm{GeV}$ to a few $\mathrm{TeV}$ are analyzed by the Erlang distribution, the inverse power-law (the Hagedorn function), and the blast-wave fit, or the superposition of two-component step function. The excitation functions of parameters such as the mean transverse momentum, initial state temperature, kinetic freeze-out temperature, and transverse flow velocity increase (slightly) from a few $\mathrm{GeV}$ to a few $\mathrm{TeV}$ and from peripheral to central collisions. At high energy and in central collisions, large collision energy is deposited in the system, which results in high degrees of excitation and expansion.
\end{abstract}

Keywords: Initial state temperature, final state temperature, Erlang distribution, inverse power-law (Hagedorn function), blast-wave fit

PACS: 12.40.Ee, 14.20.-c, 24.10.Pa, 25.75.Ag

\section{Introduction}

Temperature is an important concept in thermal and statistical physics, high energy and nuclear physics, as well as other scientific fields [1]. In high energy collisions, temperature is expected to decrease from initial state to final state due to the evolution of the interaction system. Both initial and final state temperatures are expected to obtain from particle spectra measured in experiments. In particular, the final state temperature is in fact the kinetic freeze-out temperature $\left(T_{0}\right)$ which is writhen to transverse flow velocity $\left(\beta_{T}\right)[2-4]$. Both $T_{0}$ and $\beta_{T}$ can be extracted from transverse momentum $\left(p_{T}\right)$ spectra of particles. It is expected that the initial state temperature $\left(T_{i}\right)$ can also be obtained from $p_{T}$ spectra [5].

Generally, to obtain temperatures such as $T_{i}$ and $T_{0}$ and transverse flow velocity such as $\beta_{T}$, one should describe $p_{T}$ spectra in the first place. In some cases, these parameters are related to the models or functions which are used in the fits to $p_{T}$ spectra. In other cases, these parameters are related to the types of particles due to the non-simultaneity in the process of particle emissions. These parameters are also related to collision energy, system sizes, and collision centralities. The dependences

\footnotetext{
*E-mail: fuhuliu@163.com; fuhuliu@sxu.edu.cn
}

of parameters on various factors are complex. It is very useful to find out these dependences in the understanding of collision process.

Similar to different thermometric scales or thermometers in thermal physics, one also expect a technical method to be used in the description of $p_{T}$ spectra, which should result in some parameters which are model or function independent. Obviously, the average $p_{T}\left(\left\langle p_{T}\right\rangle\right)$ and the root-mean-square $p_{T}\left(\sqrt{\left\langle p_{T}^{2}\right\rangle}\right)$ are model or function independent, which are particularly determined by experimental data themselves, though $p_{T}$ spectra in experiments are not refined in full phase space. The interested parameters are expected to relate to $\left\langle p_{T}\right\rangle$ and $\sqrt{\left\langle p_{T}^{2}\right\rangle}$.

How do particles collide in high-energy collisions? What excitation and expansion degrees of emission source can be reached in collision process? We are interested in these and related issues based on the particle $p_{T}$ spectra in experiments. It is a novel and useful method to explore the particle collision mechanism from the point of view of the initial state temperature $T_{i}$ and the final state kinetic freeze-out temperature $T_{0}$ of the emission source. Generally, transverse flow velocity $\beta_{T}$ is accompanied by $T_{0}$ in the analyses. In particular, 
both $T_{0}$ and $\beta_{T}$ are considerable at high energies.

There are various models or functions being used in the analyses of $p_{T}$ spectra. For example, in the framework of multisource thermal model [6-9], we could get the mean transverse momentum $\left\langle p_{T}\right\rangle$ of particles and the initial state temperature $T_{i}$ of emission source from fitting the $p_{T}$ spectra described by the Erlang distribution [7-9] which contains the sources number $n_{s}$ and the mean transverse momentum $\left\langle p_{t}\right\rangle$ contributed by each source. Meanwhile, we could obtain the parameter values in the Hagedorn function [10, 11] by using the same method, that is the method of fitting the $p_{T}$ spectra. In particular, both $T_{0}$ and $\beta_{T}$ can be obtained from the blast-wave fit [12-15] with remarkable coordination. Early blast-wave fit is based on Boltzmann-Gibbs statistics $[12-14]$ and the subsequent one is based on Tsallis statistics [15].

In the rest of this paper, the Erlang distribution, the Hagedorn function, and the blast-wave fits are given first in section 2. Then, in section 3, these three kinds of distributions are used to preliminarily fit the momentum and transverse momentum spectra of antiprotons produced in high energy collisions. Several representative groups of transverse momentum spectra are selected to represent and summarize the changing laws of the initial and final state temperature and other parameter values. Finally, in section 4 , we give our summary and conclusions

\section{Formalism and method}

\section{i) The Erlang distribution}

Firstly, we discuss uniformly hard and soft collision processes in the framework of the multisource thermal model [6-9]. According to the model, a given particle is produced in the collision process where a few partons have taken part in. The hard process contains two or three partons which are valence quarks. The soft process contains usually two or more partons which are gluons and sea quarks. Each (the $i$-th) parton is assumed to contribute to an exponential function $\left[f_{i}\left(p_{t}\right)\right]$ of transverse momentum $\left(p_{t}\right)$ distribution. Let $\left\langle p_{t}\right\rangle$ denotes the mean transverse momentum contributed by the $i$-th parton, we have the probability density function of $p_{t}$ to be

$$
f_{i}\left(p_{t}\right)=\frac{1}{\left\langle p_{t}\right\rangle} \exp \left(-\frac{p_{t}}{\left\langle p_{t}\right\rangle}\right)
$$

The contribution $p_{T}$ of all $n_{s}$ partons which have taken part in the collision process is the folding of $n_{s}$ exponential functions [7-9]. We have the $p_{T}$ distribution $f\left(p_{T}\right)$ (the probability density function of $p_{T}$ ) of final state particles to be the Erlang distribution

$$
f_{1}\left(p_{T}\right)=\frac{1}{N} \frac{d N}{d p_{T}}=\frac{p_{T}^{n_{s}-1}}{\left(n_{s}-1\right) !\left\langle p_{t}\right\rangle^{n}} \exp \left(-\frac{p_{T}}{\left\langle p_{t}\right\rangle}\right),
$$

which has the mean transverse momentum $\left\langle p_{T}\right\rangle=$ $n_{s}\left\langle p_{t}\right\rangle$, where $N$ denotes the number of particles and $n_{s}=2-5$ in most cases.

\section{ii) The Hagedorn function}

The Hagedorn function is generally suitable to describe the $p_{T}$ spectra of heavy flavor particles which are expectantly produced from the hard scattering process and distributed usually in a wider $p_{T}$ range. In general, the wider $p_{T}$ range is from 0 to the maximum $p_{T}$. In refs. $[10,11]$, an inverse power-law results in the probability density function of $p_{T}$ to be

$$
f_{2}\left(p_{T}\right)=\frac{1}{N} \frac{d N}{d p_{T}}=A p_{T}\left(1+\frac{p_{T}}{p_{0}}\right)^{-n}
$$

where $p_{0}$ and $n$ are the free parameters, and $A$ is the normalization constant. Eq. (3) is an empirical formula inspired by quantum chromodynamics (QCD). We also call this type of inverse power-law the Hagedorn function [10], though the inverse power-law is more famous in the community.

In some cases, Eq. (3) is possible to describe the spectra in low $p_{T}$ range which is contributed by the soft excitation process. In fact, the spectra contributed by the hard and soft processes represent sometimes similar trend due to the similarity which is widely existent in high energy collisions [16-26]. Meanwhile, Eq. (3) can be revised in different ways [27-33] which result in low probability in low $p_{T}$ or high $p_{T}$ region by using the same or similar parameters. We shall not discuss anymore the revisions of Eq. (3) to avoid trivialness.

\section{iii) The blast-wave fit}

We are also interested in the blast-wave fit with Boltzmann-Gibbs statistics in its original form. According to refs. [12-14], the blast-wave fit with BoltzmannGibbs statistics results in the probability density function of $p_{T}$ to be

$$
\begin{aligned}
f_{3}\left(p_{T}\right)= & \frac{1}{N} \frac{d N}{d p_{T}}=C_{1} p_{T} m_{T} \int_{0}^{R} r d r \times \\
& I_{0}\left[\frac{p_{T} \sinh (\rho)}{T_{0}}\right] K_{1}\left[\frac{m_{T} \cosh (\rho)}{T_{0}}\right],
\end{aligned}
$$


where $C_{1}$ is the normalized constant, $m_{T}=\sqrt{p_{T}^{2}+m_{0}^{2}}$ is the transverse mass, $m_{0}$ is the rest mass, $r$ is the radial coordinate in the thermal source, $R$ is the maximum $r$ which can be regarded as the transverse size of participant, $I_{0}$ and $K_{1}$ are the modified Bessel functions of the first and second kinds respectively, $\rho=\tanh ^{-1}[\beta(r)]$ is the boost angle, $\beta(r)=\beta_{S}(r / R)^{n_{0}}$ is a self-similar flow profile, $\beta_{S}$ is the flow velocity on the surface, and $n_{0}=2$ is used in the original form [12]. Generally, $\beta_{T}=\left(2 / R^{2}\right) \int_{0}^{R} r \beta(r) d r=2 \beta_{S} /\left(n_{0}+2\right)$.

According to ref. [15], the blast-wave fit with Tsallis statistics results in the probability density function of $p_{T}$ to be

$$
\begin{aligned}
f_{4}\left(p_{T}\right)= & \frac{1}{N} \frac{d N}{d p_{T}}=C_{2} p_{T} m_{T} \int_{-\pi}^{\pi} d \phi \int_{0}^{R} r d r\{1+ \\
& \left.\frac{q-1}{T_{0}}\left[m_{T} \cosh (\rho)-p_{T} \sinh (\rho) \cos (\phi)\right]\right\}^{-1 /(q-1)}
\end{aligned}
$$

where $C_{2}$ is the normalized constant, $q$ is an entropy index that characterizes the degree of non-equilibrium, $\phi$ denotes the azimuthal angle, and $n_{0}=1$ is used in the original form [15]. Because of $n_{0}$ being an insensitive quantity, the results corresponding to $n_{0}=1$ and 2 for the blast-wave model with Boltzmann-Gibbs or Tsallis statistics are harmonious [34]. In addition, the index $-1 /(q-1)$ used in Eq. (5) can be replaced by $-q /(q-1)$ due to $q$ being very close to 1 . This substitution results in a small and negligible difference in the distribution

As we know, the blast-wave fit with BoltzmannGibbs statistics is consistent to the fit with Tsallis statistics. In many cases, one of them is enough to use in the dada analysis. In this work, we use only the blast-wave fit with Tsallis statistics, though the fit with Boltzmann-Gibbs statistics is also usable and acceptable.

iv) Monte Carlo calculation based on $p_{T}$ distribution

Based on one of probability density functions of $p_{T}$ discussed above and the assumption of isotropic emission in sources rest frame, we can obtain other quantities and distributions. In particular, if the analytic expression is difficult to obtain, we can use the Monte Carlo method to obtain some concerned quantities, and the distributions of these concerned quantities can be obtained by statistics. These concerned quantities include, but are not limited to, momentum, energy, rapidity, velocity, and others. Conversely, the concerned $p_{T}$ and its distribution can be obtained from other distribution and the assumption of isotropic emission in sources rest frame.

In the Monte Carlo method [35], let $R_{1,2}$ denote random numbers distributed evenly in $[0,1]$. Some discrete values of $p_{T}$ can be obtained due to the following limitation

$$
\int_{0}^{p_{T}} f_{p_{T}}\left(p_{T}^{\prime}\right) d p_{T}^{\prime}<R_{1}<\int_{0}^{p_{T}+\delta p_{T}} f_{p_{T}}\left(p_{T}^{\prime}\right) d p_{T}^{\prime}
$$

where $\delta p_{T}$ denote a small shift relative to $p_{T}$. When the reference frame is transformed along the longitudinal direction ( $z$-axis), $p_{T}$ and its distribution do not changed determinately.

The change of rapidity in the transformation of reference frame should be satisfied

$$
y=y^{\prime}+R_{2}\left(y_{\max }-y_{\min }\right)+y_{\min },
$$

where $y^{\prime}$ denotes the rapidity of concerned particle in the sources rest frame, which can be obtained from the discrete values of $p_{T}$ and the assumption of isotropic emission, $y$ is the rapidity after the transformation of reference frame, and $y_{\max }$ and $y_{\min }$ are the maximum and minimum rapidity shifts of the source before the transformation of reference frame. After the transformation of reference frame, the $z$-component $\left(p_{z}\right)$ of momentum $(p)$ can be given by

$$
p_{z}=\sqrt{p_{T}^{2}+m_{0}^{2}} \sinh (y) .
$$

Naturally,

$$
p=\sqrt{p_{z}^{2}+p_{T}^{2}}
$$

According to refs. [36-38], the initial temperature is determined by

$$
T_{i}=\sqrt{\frac{\left\langle p_{T}^{2}\right\rangle}{2}} .
$$

If the $x$-component $\left(p_{x}\right)$ and $y$-component $\left(p_{y}\right)$ of $p$ are considered before or after the transformation of reference frame, we have

$$
T_{i}=\sqrt{\left\langle p_{x}^{2}\right\rangle}=\sqrt{\left\langle p_{y}^{2}\right\rangle} .
$$

Obviously, $T_{i}$ is invariant in the transformation of reference frame. In the sources rest frame, if the $z$ component of momentum is $p_{z}^{\prime}$, we also have

$$
T_{i}=\sqrt{\left\langle p_{z}^{\prime 2}\right\rangle}
$$

due to the assumption of isotropic emission. 

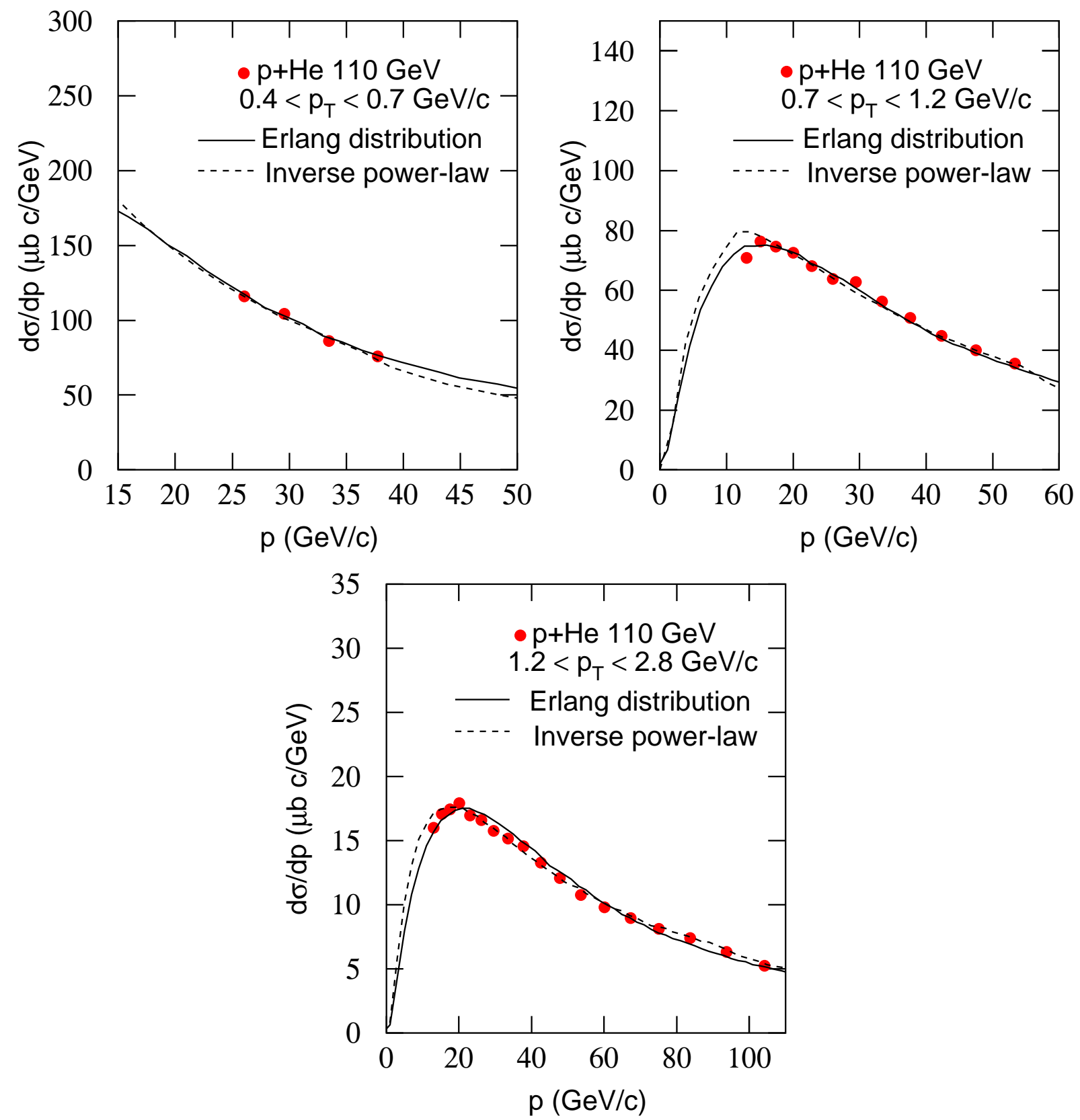

Fig. 1. Antiproton production differential cross-section as a function of momentum, integrated over various transverse momentum ranges, in $p+\mathrm{He}$ collisions at $110 \mathrm{GeV}$. The data represent the results of LHCb Collaboration [39]. The solid and dashed curves represent the results fitted by the Erlang distribution Eq. (2) and the inverse power-law Eq. (3), where the Monte Carlo calculation is used to transform transverse momenta to momenta.

\section{Results and discussion}

Figure 1 shows the momentum spectra, $d \sigma / d p$, of antiprotons $(\bar{p})$ produced in proton induced helium $(p+\mathrm{He})$ collisions at center-of-mass energy per nucleon pair $\sqrt{s_{N N}}=110 \mathrm{GeV}$, where $\sigma$ denotes the cross-section. Corresponding to the left-upper, right-upper, and lower panels, the values of $p_{T}$ are limited in $0.4-0.7,0.7-1.2$, and $1.2-2.8 \mathrm{GeV} / c$, respectively. The circles presented in the three panels represent the experimental data of $\bar{p}$ measured by the LHCb Collaboration [39]. The data points are fitted by the Erlang distribution (the solid curves) and the inverse power-law (the dashed curves) respectively, which are obtained by using the Monte Carlo calculation based on Eqs. (2) and (3) respectively. In the calculations, the method of least square is used 
Table 1. Values of $\left\langle p_{T}\right\rangle, p_{0}, n$, and $\chi^{2} /$ dof corresponding to the solid and dashed curves in Figs. 1 and 2, where the values of $\chi^{2} /$ dof for the solid and dashed curves are listed in terms of value $_{1} /$ value $_{2}$.

\begin{tabular}{|c|c|c|c|c|c|}
\hline Figure & Range of $p\left(p_{T}\right)(\mathrm{GeV} / c)$ & $\left\langle p_{T}\right\rangle(\mathrm{GeV} / c)$ & $p_{0}(\mathrm{GeV} / c)$ & $\frac{n}{n}$ & $\chi^{2} /$ dof \\
\hline \multirow{3}{*}{ Figure 1} & $0.4-0.7$ & $3.000 \pm 0.020$ & $56.30 \pm 0.018$ & $12.30 \pm 0.30$ & $0.597 / 0.981$ \\
\hline & $0.7-1.2$ & $4.500 \pm 0.013$ & $14.50 \pm 0.010$ & $4.20 \pm 0.10$ & $1.166 / 3.017$ \\
\hline & $1.2-2.8$ & $6.460 \pm 0.020$ & $9.80 \pm 0.024$ & $5.00 \pm 0.10$ & $0.994 / 1.489$ \\
\hline \multirow[t]{17}{*}{$\overline{\text { Figure } 2}$} & $12.0-14.0$ & $0.961 \pm 0.005$ & $9.00 \pm 0.02$ & $48.00 \pm 0.01$ & $0.257 / 0.628$ \\
\hline & $14.0-16.2$ & $0.973 \pm 0.006$ & $9.00 \pm 0.01$ & $48.00 \pm 0.01$ & $0.125 / 0.252$ \\
\hline & $16.2-18.7$ & $0.986 \pm 0.006$ & $9.00 \pm 0.02$ & $48.00 \pm 0.01$ & $0.100 / 0.845$ \\
\hline & $18.7-21.4$ & $1.003 \pm 0.008$ & $9.00 \pm 0.02$ & $48.00 \pm 0.01$ & $0.210 / 0.572$ \\
\hline & $\begin{array}{l}21.4-24.4 \\
24.4-27.7\end{array}$ & $\begin{array}{l}1.011 \pm 0.004 \\
0.943+0.005\end{array}$ & $9.00 \pm 0.01$ & $\begin{array}{l}48.00 \pm 0.01 \\
48.00+0.01\end{array}$ & $0.213 / 0.270$ \\
\hline & $\begin{array}{l}27.7-31.4 \\
27.4\end{array}$ & $\begin{array}{l}0.943 \pm 0.005 \\
0.943 \pm 0.010\end{array}$ & $\begin{array}{l}9.00 \pm 0.01 \\
9.00 \pm 0.02\end{array}$ & $\begin{array}{l}48.00 \pm 0.01 \\
48.00 \pm 0.01\end{array}$ & $\begin{array}{l}0.090 / 0.734 \\
0.134 / 0.733\end{array}$ \\
\hline & $31.4-35.5$ & $0.943 \pm 0.008$ & $9.00 \pm 0.02$ & $48.00 \pm 0.01$ & $0.376 / 0.236$ \\
\hline & $35.5-40.0$ & $0.987 \pm 0.007$ & $9.00 \pm 0.01$ & $48.00 \pm 0.01$ & $0.196 / 0.098$ \\
\hline & $40.0-45.0$ & $1.109 \pm 0.005$ & $9.00 \pm 0.01$ & $48.00 \pm 0.02$ & $0.221 / 0.073$ \\
\hline & $45.0-50.5$ & $1.109 \pm 0.010$ & $9.00 \pm 0.02$ & $48.00 \pm 0.02$ & $0.133 / 0.084$ \\
\hline & $50.5-56.7$ & $1.199 \pm 0.010$ & $9.00 \pm 0.01$ & $48.00 \pm 0.01$ & $0.063 / 0.037$ \\
\hline & $56.7-63.5$ & $1.288 \pm 0.015$ & $9.00 \pm 0.02$ & $48.00 \pm 0.01$ & $0.136 / 0.053$ \\
\hline & $63.5-71.0$ & $1.301 \pm 0.015$ & $9.00 \pm 0.01$ & $48.00 \pm 0.01$ & $0.162 / 0.106$ \\
\hline & $71.0-79.3$ & $1.407 \pm 0.020$ & $9.00 \pm 0.01$ & $48.00 \pm 0.01$ & $0.113 / 0.125$ \\
\hline & $79.3-88.5$ & $1.541 \pm 0.020$ & $9.00 \pm 0.02$ & $48.00 \pm 0.01$ & $0.113 / 0.153$ \\
\hline & $88.5-98.7$ & $1.729 \pm 0.020$ & $9.00 \pm 0.01$ & $48.00 \pm 0.01$ & $0.190 / 0.065$ \\
\hline & $98.7-110.0$ & $1.739 \pm 0.015$ & $9.00 \pm 0.01$ & $48.00 \pm 0.01$ & $0.225 / 0.425$ \\
\hline
\end{tabular}


Table 2. Values of $\left\langle p_{T}\right\rangle, T_{i}, T_{0}, \beta_{T}, p_{0}$, and $n$ corresponding to the curves in Figs. $3-5$. The values presented in terms of value ${ }_{1} /$ value $_{2}$ denote respectively the parameters of the first and second components. In all cases, $q=1.01$ which is not listed in the table. The values of $\chi^{2} /$ dof are in normal range and not listed in the table to reduce its length

\begin{tabular}{|c|c|c|c|c|c|c|c|}
\hline 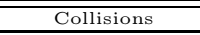 & 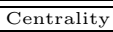 & $\overline{\left\langle p_{T}\right\rangle(\mathrm{GeV} / c)}$ & $\overline{T T_{i}(\mathrm{GeV})}$ & $\overline{T T_{0}(\mathrm{GeV})}$ & $\overline{\overline{\beta_{T}(c)}}$ & $\overline{p_{0}(\mathrm{GeV} / c)}$ & $\bar{n}$ \\
\hline \multirow{7}{*}{$7.7 \mathrm{GeV} \mathrm{Au}+\mathrm{Au}$} & $0-5 \%$ & $0.785 \pm 0.023$ & $0.579 \pm 0.018$ & $0.135 \pm 0.002$ & $0.340 \pm 0.020$ & $3.80 \pm 0.20$ & $11.10 \pm 0.40$ \\
\hline & $5-10 \%$ & $0.777 \pm 0.013$ & $0.573 \pm 0.010$ & $0.133 \pm 0.003$ & $0.325 \pm 0.025$ & $3.60 \pm 0.30$ & $11.25 \pm 0.75$ \\
\hline & $10-20 \%$ & $0.762 \pm 0.030$ & $0.562 \pm 0.024$ & $0.126 \pm 0.003$ & $0.295 \pm 0.015$ & $3.55 \pm 0.20$ & $12.50 \pm 0.50$ \\
\hline & $20-30 \%$ & $0.754 \pm 0.025$ & $0.556 \pm 0.020$ & $0.125 \pm 0.003$ & $0.285 \pm 0.015$ & $3.47 \pm 0.35$ & $13.00 \pm 1.00$ \\
\hline & $30-40 \%$ & $0.732 \pm 0.020$ & $0.541 \pm 0.016$ & $0.122 \pm 0.004$ & $0.255 \pm 0.015$ & $3.35 \pm 0.35$ & $13.50 \pm 0.50$ \\
\hline & $40-$ & & $0.530 \pm 0.014$ & $0.116 \pm 0.003$ & $0.235 \pm 0.015$ & $3.25 \pm 0.20$ & $14.30 \pm 0.70$ \\
\hline & $\begin{array}{l}60-70 \% \\
70-80 \%\end{array}$ & & $0.425 \pm 0.018$ & $\begin{array}{l}0.106 \pm 0.004 \\
0.096 \pm 0.004\end{array}$ & $\begin{array}{l}0.165 \pm 0.015 \\
0.145 \pm 0.015\end{array}$ & $\begin{array}{l}3.00 \pm 0.10 \\
2.90 \pm 0.20\end{array}$ & \\
\hline \multirow{7}{*}{$11.5 \mathrm{GeV} \mathrm{Au}+\mathrm{Au}$} & $0-5 \%$ & $0.728 \pm 0.020 / 1.318 \pm 0.105$ & $0.530 \pm 0.015 / 0.945 \pm 0.035$ & $0.185 \pm 0.005 / 0.113 \pm 0.013$ & $0.315 \pm 0.005 / 0.280 \pm 0.010$ & $4.30 \pm 0.20 / 3.10 \pm 0.10$ & $8.50 \pm 0.50 / 16.00 \pm 0.50$ \\
\hline & $5-10 \%$ & $0.722 \pm 0.031 / 1.319 \pm 0.098$ & $0.526 \pm 0.018 / 0.945 \pm 0.025$ & $0.180 \pm 0.015 / 0.108 \pm 0.012$ & $0.314 \pm 0.010 / 0.275 \pm 0.020$ & $4.25 \pm 0.20 / 3.10 \pm 0.10$ & $9.00 \pm 0.50 / 16.50 \pm 0.50$ \\
\hline & $10-20 \%$ & $0.723 \pm 0.028 / 1.312 \pm 0.151$ & $0.527 \pm 0.030 / 0.940 \pm 0.050$ & $0.170 \pm 0.010 / 0.105 \pm 0.005$ & $0.314 \pm 0.010 / 0.268 \pm 0.013$ & $4.20 \pm 0.30 / 3.10 \pm 0.10$ & $10.00 \pm 0.50 / 17.00 \pm 0.50$ \\
\hline & $20-30 \%$ & $0.716 \pm 0.018 / 1.298 \pm 0.168$ & $0.515 \pm 0.025 / 0.929 \pm 0.085$ & $0.127 \pm 0.010$ & $0.255 \pm 0.010$ & $4.00 \pm 0.30 / 3.10 \pm 0.20$ & $11.00 \pm 0.50 / 17.00 \pm 0.50$ \\
\hline & $30-40 \%$ & $0.701 \pm 0.020 / 1.277 \pm 0.155$ & $0.510 \pm 0.040 / 0.911 \pm 0.082$ & $0.124 \pm 0.010$ & $0.240 \pm 0.010$ & $70 \pm 0.30 / 3.10 \pm 0.30$ & $.15 \pm 0.50 / 17.50 \pm 0.50$ \\
\hline & $50-60 \%$ & $\begin{array}{l}0.697 \pm 0.010 \\
0.679+0.010\end{array}$ & $\begin{array}{l}0.531 \pm 0.018 \\
0.504+0.018\end{array}$ & $\begin{array}{l}0.113 \pm 0.008 \\
0.110 \pm 0.008\end{array}$ & $\begin{array}{l}0.218 \pm 0.012 \\
0.205 \pm 0.015\end{array}$ & $3.30 \pm 0.20 / 3.20 \pm 0.10$ & $12.75 \pm 0.75 / 18.20 \pm 0.20$ \\
\hline & $\begin{array}{l}60-70 \% \\
70-80 \%\end{array}$ & $\begin{array}{l}0.679 \pm 0.010 \\
0.656 \pm 0.010\end{array}$ & $\begin{array}{l}0.504 \pm 0.018 \\
0.483 \pm 0.018\end{array}$ & $\begin{array}{l}0.110 \pm 0.008 \\
0.105 \pm 0.008\end{array}$ & $\begin{array}{l}0.205 \pm 0.015 \\
0.185 \pm 0.015\end{array}$ & $\begin{array}{l}3.20 \pm 0.10 \\
3.00 \pm 0.10\end{array}$ & $\begin{array}{l}15.90 \pm 0.30 \\
16.25 \pm 0.75\end{array}$ \\
\hline \multirow{8}{*}{$19.6 \mathrm{Gev} \mathrm{Au+Au}$} & $0-5 \%$ & $0.735 \pm 0.021 / 1.336 \pm 0.125$ & $0.535 \pm 0.015 / 0.958 \pm 0.037$ & $0.190 \pm 0.010 / 0.110 \pm 0.010$ & $0.330 \pm 0.010 / 0.300 \pm 0.020$ & $4.50 \pm 0.20 / 3.40 \pm 0.20$ & $9.00 \pm 0.50 / 16.50 \pm 0.50$ \\
\hline & $5-10 \%$ & $0.727 \pm 0.020 / 1.326 \pm 0.124$ & $0.530 \pm 0.010 / 0.949 \pm 0.030$ & $0.185 \pm 0.010 / 0.107 \pm 0.008$ & $315 \pm 0.005 / 0.300 \pm 0$ & $4.40 \pm 0.20 / 3.30 \pm 0.20$ & $9.50 \pm 1.00 / 17.40 \pm 0.60$ \\
\hline & $10-20 \%$ & $0.721 \pm 0.010 / 1.325 \pm 0.125$ & $0.525 \pm 0.015 / 0.950 \pm 0.040$ & $0.184 \pm 0.010 / 0.105 \pm 0$ & $0.313 \pm 0.010 / 0.300 \pm 0$ & $4.20 \pm 0.20 / 3.40 \pm 0.20$ & $10.50 \pm 0.50 / 17.40 \pm 0.60$ \\
\hline & $20-30 \%$ & $0.719 \pm 0.019 / 1.324 \pm 0.176$ & $0.523 \pm 0.013 / 0.950 \pm 0.080$ & $0.132 \pm 0.004$ & $0.280 \pm 0.010$ & $4.00 \pm 0.40 / 3.30 \pm 0.20$ & $11.10 \pm 0.50 / 17.40 \pm 0.90$ \\
\hline & & $0.700 \pm 0.100 / 1.323 \pm 0$ & $10 \pm 0.045 / 0.949 \pm 0.079$ & $0.128 \pm 0.003$ & $0.255 \pm 0.005$ & $3.80 \pm 0.20 / 3.20 \pm 0.30$ & $12.00 \pm 0.80 / 17.80 \pm 0.60$ \\
\hline & $40-50 \%$ & $0.694 \pm 0.090 / 1.319 \pm 0.209$ & $0.506 \pm 0.072 / 0.945 \pm 0$ & \pm 0.003 & $0.253 \pm 0.005$ & $0 \pm 0.20$ & $2.60 \pm 0.50 / 17.80 \pm 0.20$ \\
\hline & $50-60 \%$ & $0.737 \pm 0.010$ & $0.569 \pm 0.018$ & $0.118 \pm 0.003$ & $0.235 \pm 0.005$ & $3.40 \pm 0.20 / 3.00 \pm 0.10$ & $12.60 \pm 0.40 / 18.20 \pm 0.50$ \\
\hline & $\begin{array}{l}60-70 \% \\
70-80 \%\end{array}$ & $\begin{array}{l}0.705 \pm 0.015 \\
0.676 \pm 0.015\end{array}$ & $\begin{array}{l}0.542 \pm 0.014 \\
0.516 \pm 0.014\end{array}$ & $\begin{array}{l}0.113 \pm 0.003 \\
0.110 \pm 0.002\end{array}$ & $\begin{array}{l}0.205 \pm 0.005 \\
0.185 \pm 0.005\end{array}$ & $3.40 \pm 0.20 / 3.00 \pm 0.20$ & $12.90 \pm 0.90 / 19.00 \pm 0.30$ \\
\hline \multirow{6}{*}{$27 \mathrm{GeV} \mathrm{Au}+\mathrm{Au}$} & $0-5 \%$ & $0.726 \pm 0.028 / 1.328 \pm 0.153$ & $\begin{array}{c}0.516 \pm 0.014 \\
0.528 \pm 0.015 / 0.954 \pm 0.052\end{array}$ & $\frac{0.110 \pm 0.002}{0.145 \pm 0.003}$ & $\frac{0.185 \pm 0.005}{0.300 \pm 0.005}$ & $\frac{3.10 \pm 0.10}{4.90 \pm 0.20 / 3.90 \pm 0.20}$ & $\begin{array}{c}16.90 \pm 0.50 \\
10.50 \pm 1.20 / 16.50 \pm 0.50\end{array}$ \\
\hline & $5-10 \%$ & $0.720 \pm 0.022 / 1.329 \pm 0$. & $0.523 \pm 0.019 / 0.955 \pm 0$. & $0.142 \pm 0.003$ & $0.300 \pm 0.005$ & $4.70 \pm 0.20 / 3.60 \pm 0.20$ & $10.50 \pm 1.00 / 16.50 \pm 0.50$ \\
\hline & $10-$ & $\pm 0.029 / 1.323 \pm 0.143$ & $0.522 \pm 0.011 / 0.950 \pm 0$. & \pm 0.002 & $0.295 \pm 0.005$ & $4.50 \pm 0.20$ & $11.25 \pm 0.50 / 16.50 \pm 0.50$ \\
\hline & $40-50 \%$ & $0.706 \pm 0.065 / 1.296 \pm 0.250$ & $0.513 \pm 0.040 / 0.930 \pm 0.085$ & $0.128 \pm 0.003$ & $0.263 \pm 0.003$ & $3.90 \pm 0.20 / 3.30 \pm 0.20$ & $13.00 \pm 0.50 / 17.70 \pm 0.30$ \\
\hline & $50-60 \%$ & $0.760 \pm 0.020$ & \pm 0.018 & $0.125 \pm 0.003$ & $0.225 \pm 0.003$ & $3.90 \pm 0.20 / 3.20 \pm 0.10$ & $13.00 \pm 0.30 / 18.20 \pm 0.40$ \\
\hline & $\begin{array}{l}60-70 \% \\
70-80 \%\end{array}$ & $=0.025$ & $\begin{array}{l}0.564 \pm 0.014 \\
0.521 \pm 0.014\end{array}$ & $\begin{array}{l}0.123 \pm 0.003 \\
0.118 \pm 0.002\end{array}$ & $\begin{array}{l}0.205 \pm 0.005 \\
0.179 \pm 0.003\end{array}$ & $\begin{array}{c}3.70 \pm 0.30 / 3.00 \pm 0.20 \\
3.20 \pm 0.10\end{array}$ & $\begin{array}{c}14.00 \pm 0.50 / 18.90 \pm 0.70 \\
11.50 \pm 0.50\end{array}$ \\
\hline \multirow{8}{*}{$39 \mathrm{GeV} \mathrm{Au}+\mathrm{Au}$} & $\frac{0-80 \%}{0-5 \%}$ & $\frac{0.722 \pm 0.022}{0.740 \pm 0.021 / 1.352 \pm 0.1}$ & $38 \pm 0.01$ & $195 \pm 0.0$ & $0.360 \pm 0$ & $5.30 \pm 0$. & $17.00 \pm 1.00$ \\
\hline & & \pm 0 . & \pm 0 . & \pm 0 & \pm 0 & \pm 0 & $9.00 \pm 0$ \\
\hline & & \pm 0 . & \pm 0 & $0.193 \pm 0$. & $0.350 \pm 0$. & $4.90 \pm 0$. & $10.00 \pm 0.50 / 17.00 \pm 1.00$ \\
\hline & $\%$ & $9 \pm 0.156$ & \pm 0.0 & & 0.2 & $4.70 \pm 0$ & $1.00 \pm 0.50 / 17.50 \pm 1.00$ \\
\hline & $30-40 \%$ & $0.718 \pm 0.010 / 1.329 \pm 0.118$ & $0.523 \pm 0.013 / 0.953 \pm 0.040$ & $0.135 \pm 0.002$ & $0.283 \pm 0.003$ & $4.50 \pm 0.30 / 3.60 \pm 0.20$ & $11.25 \pm 0.75 / 17.40 \pm 0.60$ \\
\hline & $40-50 \%$ & $0.837 \pm 0.040$ & $0.600 \pm 0.022$ & $0.133 \pm 0.003$ & $0.255 \pm 0.005$ & & $13.50 \pm 0.50 / 17.50 \pm 1.00$ \\
\hline & 50 & $0.813 \pm 0.020$ & $\begin{array}{l}0.580 \pm 0.025 \\
0.569 \pm 0.022\end{array}$ & $0.130 \pm 0.003$ & $\begin{array}{l}0.233 \pm 0.003 \\
0.213 \pm 0.003\end{array}$ & $\begin{array}{l}4.00 \pm 0.20 / 3.20 \pm 0.10 \\
3.80\end{array}$ & $14.20 \pm 0.40 / 18.30 \pm 0.30$ \\
\hline & $\begin{array}{l}60-70 \% \\
70-80 \%\end{array}$ & $\begin{array}{l}0.776 \pm 0.025 \\
0.750 \pm 0.022\end{array}$ & $\begin{array}{l}0.569 \pm 0.022 \\
0.547 \pm 0.028\end{array}$ & $\begin{array}{l}0.128 \pm 0.002 \\
0.125 \pm 0.002\end{array}$ & $\begin{array}{l}0.213 \pm 0.003 \\
0.193 \pm 0.003\end{array}$ & $3.38 \pm 0.13$ & $17.50 \pm 0.20$ \\
\hline
\end{tabular}


Table 2. Continued.

\begin{tabular}{|c|c|c|c|c|c|c|c|}
\hline $\begin{array}{ll}\text { Collisions } \\
\end{array}$ & Centrality & $\overline{\left\langle\left\langle p_{T}\right\rangle(\mathrm{GeV} / c)\right.}$ & 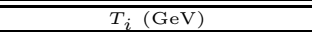 & $T_{0}(\mathrm{GeV})$ & $\beta_{T}(c)$ & $\frac{p_{0}(\mathrm{GeV} / c)}{1}$ & $\bar{n}$ \\
\hline \multirow{9}{*}{$62.4 \mathrm{GeV} \mathrm{Au}+\mathrm{Au}$} & $0-5 \%$ & $1.130 \pm 0.025$ & $0.856 \pm 0.020$ & $0.210 \pm 0.010$ & $0.425 \pm 0.010$ & $8.10 \pm 0.30 / 11.00 \pm 0.50$ & $6.00 \pm 0.50 / 21.00 \pm 1.00$ \\
\hline & 5-10\% & $1.110 \pm 0.015$ & $0.842 \pm 0.012$ & $0.205 \pm 0.005$ & $0.405 \pm 0.010$ & $7.80 \pm 0.30 / 10.50 \pm 0.50$ & $7.00 \pm 0.50 / 21.00 \pm 1.00$ \\
\hline & $10-20 \%$ & $1.104 \pm 0.015$ & $0.839 \pm 0.012$ & $0.195 \pm 0.005$ & $0.395 \pm 0.005$ & $7.50 \pm 0.30 / 10.50 \pm 0.30$ & $8.00 \pm 0.30 / 21.00 \pm 1.00$ \\
\hline & $20-30 \%$ & $1.063 \pm 0.035$ & $0.811 \pm 0.029$ & $0.178 \pm 0.003$ & $0.370 \pm 0.010$ & $7.20 \pm 0.30 / 10.00 \pm 0.50$ & $9.00 \pm 0.50 / 22.00 \pm 2.00$ \\
\hline & $30-40 \%$ & $1.022 \pm 0.035$ & $0.782 \pm 0.029$ & $0.162 \pm 0.004$ & $0.350 \pm 0.010$ & $6.80 \pm 0.40 / 9.00 \pm 0.50$ & $10.00 \pm 0.50 / 22.50 \pm 1.50$ \\
\hline & & & $0.758 \pm 0.012$ & $0.155 \pm 0.005$ & $0.310 \pm 0.010$ & $6.50 \pm 0.30 / 7.60 \pm 0.40$ & $11.00 \pm 0.50 / 22.00 \pm$ \\
\hline & $50-60 \%$ & $0.933 \pm 0.010$ & $0.718 \pm 0.008$ & $0.150 \pm 0.002$ & $0.295 \pm 0.005$ & $6.65 \pm 0.25$ & $16.50 \pm 0.50$ \\
\hline & $60-70 \%$ & $0.871 \pm 0.015$ & $0.671 \pm 0.012$ & $0.147 \pm 0.002$ & $0.265 \pm 0.005$ & $6.30 \pm 0.30$ & $17.75 \pm 0.75$ \\
\hline & $70-80 \%$ & $0.840 \pm 0.012$ & $0.648 \pm 0.013$ & $0.143 \pm 0.003$ & $0.215 \pm 0.005$ & $6.00 \pm 0.30$ & $18.50 \pm 1.00$ \\
\hline \multirow{7}{*}{$130 \mathrm{GeV} \mathrm{Au}+\mathrm{Au}$} & $0-6 \%$ & $1.139 \pm 0.025$ & $0.862 \pm 0.020$ & $0.220 \pm 0.010$ & $0.435 \pm 0.010$ & $11.70 \pm 0.30$ & $13.50 \pm 1.50$ \\
\hline & $6-11 \%$ & $1.125 \pm 0.025$ & $0.853 \pm 0.020$ & $0.210 \pm 0.005$ & $0.410 \pm 0.010$ & $11.20 \pm 0.30$ & $13.70 \pm 1.50$ \\
\hline & $12-18 \%$ & $1.115 \pm 0.015$ & $0.846 \pm 0.012$ & $0.198 \pm 0.005$ & $0.398 \pm 0.008$ & $10.70 \pm 0.30$ & $13.90 \pm 1.10$ \\
\hline & $18-26 \%$ & $1.104 \pm 0.015$ & $0.839 \pm 0.012$ & $0.187 \pm 0.007$ & $0.380 \pm 0.005$ & $10.35 \pm 0.50$ & $14.20 \pm 2.00$ \\
\hline & $26-34 \%$ & $1.089 \pm 0.020$ & $0.828 \pm 0.012$ & $0.165 \pm 0.005$ & $0.365 \pm 0.010$ & $10.15 \pm 0.20$ & $15.50 \pm 1.30$ \\
\hline & $34-45 \%$ & $1.051 \pm 0.015$ & $0.802 \pm 0.012$ & $0.160 \pm 0.005$ & $0.360 \pm 0.010$ & $10.00 \pm 0.40$ & $16.50 \pm 1.50$ \\
\hline & $\begin{array}{l}45-58 \% \\
58-85 \%\end{array}$ & $\begin{array}{l}1.001 \pm 0.015 \\
0.941 \pm 0.020\end{array}$ & $\begin{array}{l}0.767 \pm 0.012 \\
0.724 \pm 0.010\end{array}$ & $\begin{array}{l}0.150 \pm 0.005 \\
0.143 \pm 0.003\end{array}$ & $\begin{array}{l}0.350 \pm 0.010 \\
0.315 \pm 0.005\end{array}$ & $\begin{array}{l}9.00 \pm 0.20 \\
8.50 \pm 0.20\end{array}$ & $\begin{array}{l}17.80 \pm 1.30 \\
19.50+1.20\end{array}$ \\
\hline \multirow{8}{*}{$200 \mathrm{GeV} \mathrm{Au}+\mathrm{Au}$} & $0-5 \%$ & $\frac{0.041 \pm 0.010}{1.141 \pm 0.010}$ & $\frac{0.124 \pm 0.010}{0.864 \pm 0.008}$ & $\frac{0.143 \pm 0.003}{0.220 \pm 0.020}$ & $\frac{0.315 \pm 0.005}{0.435 \pm 0.010}$ & $\frac{8.50 \pm 0.20}{12.60 \pm 0.30 / 12.00 \pm 0.50}$ & $\frac{19.0 \pm \pm 1.20}{10.00 \pm 0.50 / 18.00 \pm 2.00}$ \\
\hline & $5-10 \%$ & $1.125 \pm 0.015$ & $0.854 \pm 0.012$ & $0.210 \pm 0.010$ & $0.415 \pm 0.010$ & $12.30 \pm 0.30 / 11.00 \pm 0.50$ & $11.00 \pm 2.00 / 18.00 \pm 5.00$ \\
\hline & $10-20 \%$ & $1.118 \pm 0.025$ & $0.848 \pm 0.020$ & $0.200 \pm 0.015$ & $0.410 \pm 0.015$ & $12.00 \pm 0.30 / 10.50 \pm 0.50$ & $12.50 \pm 0.50 / 18.00 \pm 2.00$ \\
\hline & $20-30 \%$ & $1.095 \pm 0.025$ & $0.832 \pm 0.020$ & $0.190 \pm 0.025$ & $0.385 \pm 0.020$ & $10.20 \pm 0.30$ & $15.50 \pm 1.00$ \\
\hline & $30-40 \%$ & $1.055 \pm 0.025$ & $0.805 \pm 0.101$ & $0.167 \pm 0.015$ & $0.365 \pm 0.010$ & $9.90 \pm 0.30$ & $17.00 \pm 0.50$ \\
\hline & $40-50 \%$ & $1.017 \pm 0.020$ & $0.779 \pm 0.016$ & $0.162 \pm 0.015$ & $0.335 \pm 0.005$ & $9.60 \pm 0.30$ & $18.50 \pm 0.50$ \\
\hline & $50-60 \%$ & $0.983 \pm 0.010$ & $0.754 \pm 0.018$ & $0.156 \pm 0.003$ & $0.335 \pm 0.005$ & $9.30 \pm 0.30$ & $20.00 \pm 0.50$ \\
\hline & $\begin{array}{l}60-70 \% \\
70-80 \% \\
\end{array}$ & $\begin{array}{l}0.908 \pm 0.012 \\
0.881 \pm 0.012\end{array}$ & $\begin{array}{l}0.699 \pm 0.014 \\
0.679 \pm 0.013\end{array}$ & $\begin{array}{l}0.150 \pm 0.003 \\
0.145 \pm 0.006\end{array}$ & $\begin{array}{l}0.285 \pm 0.005 \\
0.265 \pm 0.005\end{array}$ & $\begin{array}{l}9.00 \pm 0.30 \\
8.80 \pm 0.30\end{array}$ & $\begin{array}{l}23.00 \pm 0.30 \\
24.00 \pm 0.30\end{array}$ \\
\hline \multirow{3}{*}{$200 \mathrm{GeV} d+\mathrm{Au}$} & $0-20 \%$ & $0.972 \pm 0.015$ & $0.741 \pm 0.013$ & $0.163 \pm 0.006$ & $0.287 \pm 0.006$ & $9.60 \pm 0.50$ & $22.50 \pm 2.00$ \\
\hline & $20-40 \%$ & $0.965 \pm 0.010$ & $0.736 \pm 0.009$ & $0.153 \pm 0.006$ & $0.270 \pm 0.006$ & $9.30 \pm 0.50$ & $23.00 \pm 1.50$ \\
\hline & $40-100 \%$ & $0.924 \pm 0.010$ & $0.705 \pm 0.009$ & $0.148 \pm 0.007$ & $0.250 \pm 0.007$ & $9.00 \pm 0.50$ & $24.50 \pm 1.50$ \\
\hline \multirow[t]{9}{*}{$2.76 \mathrm{TeV} \mathrm{Pb}+\mathrm{Pb}$} & $\begin{array}{l}0-5 \% \\
5-50 \%\end{array}$ & $0.889 \pm 0.023 / 1.957 \pm 0.142$ & $\begin{array}{l}0.659 \pm 0.031 / 1.439 \pm 0.065 \\
0656 \pm 0.0201 .439 \pm 0.043\end{array}$ & $\begin{array}{l}0.310 \pm 0.060 / 0.170 \pm 0.010 \\
0.280 \pm 0.0500170 \pm 0.005\end{array}$ & $\begin{array}{l}0.500 \pm 0.010 / 0.371 \pm 0.005 \\
0.500+0.0100370 \pm 0.005\end{array}$ & $\begin{array}{l}22.50 \pm 1.50 / 11.00 \pm 0.10 \\
22.00+200 / 10.60+0.20\end{array}$ & $\begin{array}{l}18.00 \pm 1.00 / 26.00 \pm 0.10 \\
20000+1.0026 .30+0.20\end{array}$ \\
\hline & $10-20 \%$ & $0.879 \pm 0.051 / 1.956 \pm 0.230$ & $0.653 \pm 0.025 / 1.439 \pm 0.071$ & $\begin{array}{l}0.280 \pm 0.050 / 0.170 \pm 0.005 \\
0.250 \pm 0.020 / 0.165 \pm 0.010\end{array}$ & $\begin{array}{l}0.500 \pm 0.010 / 0.370 \pm 0.005 \\
0.480\end{array} 0.010 / 0.370 \pm 0.005$ & $\begin{array}{l}22.00 \pm 2.000 / 10.60 \pm 0.20 \\
18.50+1.00 / 10.70+0.30\end{array}$ & $\begin{array}{l}20.00 \pm 1.00 / 26.30 \pm 0.20 \\
20.50 \pm 1.50 / 26.50 \pm 0.50\end{array}$ \\
\hline & $20-30 \%$ & $0.862 \pm 0.080 / 1.955 \pm 0.450$ & $0.642 \pm 0.023 / 1.439 \pm 0.038$ & $0.220 \pm 0.030 / 0.165 \pm 0.008$ & $0.450 \pm 0.010 / 0.365 \pm 0.005$ & $17.00 \pm 1.00 / 10.50 \pm 0.50$ & $21.50 \pm 1.00 / 26.80 \pm 0.80$ \\
\hline & $30-40 \%$ & $0.857 \pm 0.058 / 1.944 \pm 0.500$ & $0.638 \pm 0.015 / 1.428 \pm 0.027$ & $0.200 \pm 0.020 / 0.165 \pm 0.010$ & $0.450 \pm 0.010 / 0.360 \pm 0.007$ & $16.00 \pm 0.80 / 10.00 \pm 0.40$ & $23.50 \pm 1.50 / 27.00 \pm 0.50$ \\
\hline & $40-50 \%$ & $1.110 \pm 0.120$ & $0.918 \pm 0.039$ & $0.180 \pm 0.001$ & $0.370 \pm 0.001$ & $11.70 \pm 0.20$ & $26.50 \pm 0.50$ \\
\hline & $50-60 \%$ & $1.104 \pm 0.111$ & $0.906 \pm 0.031$ & $0.170 \pm 0.005$ & $0.360 \pm 0.005$ & $11.50 \pm 0.20$ & $27.00 \pm 0.20$ \\
\hline & $60-70 \%$ & $1.050 \pm 0.110$ & $0.860 \pm 0.031$ & $0.163 \pm 0.003$ & $0.350 \pm 0.005$ & $11.20 \pm 0.30$ & $27.50 \pm 0.50$ \\
\hline & $70-80 \%$ & $1.033 \pm 0.110$ & $0.845 \pm 0.031$ & $0.155 \pm 0.005$ & $0.340 \pm 0.010$ & $11.00 \pm 0.50$ & $28.00 \pm 1.00$ \\
\hline & $80-90 \%$ & $0.961 \pm 0.090$ & $0.782 \pm 0.030$ & $0.150 \pm 0.005$ & $0.336 \pm 0.006$ & $10.50 \pm 0.50$ & $29.00 \pm 1.00$ \\
\hline
\end{tabular}



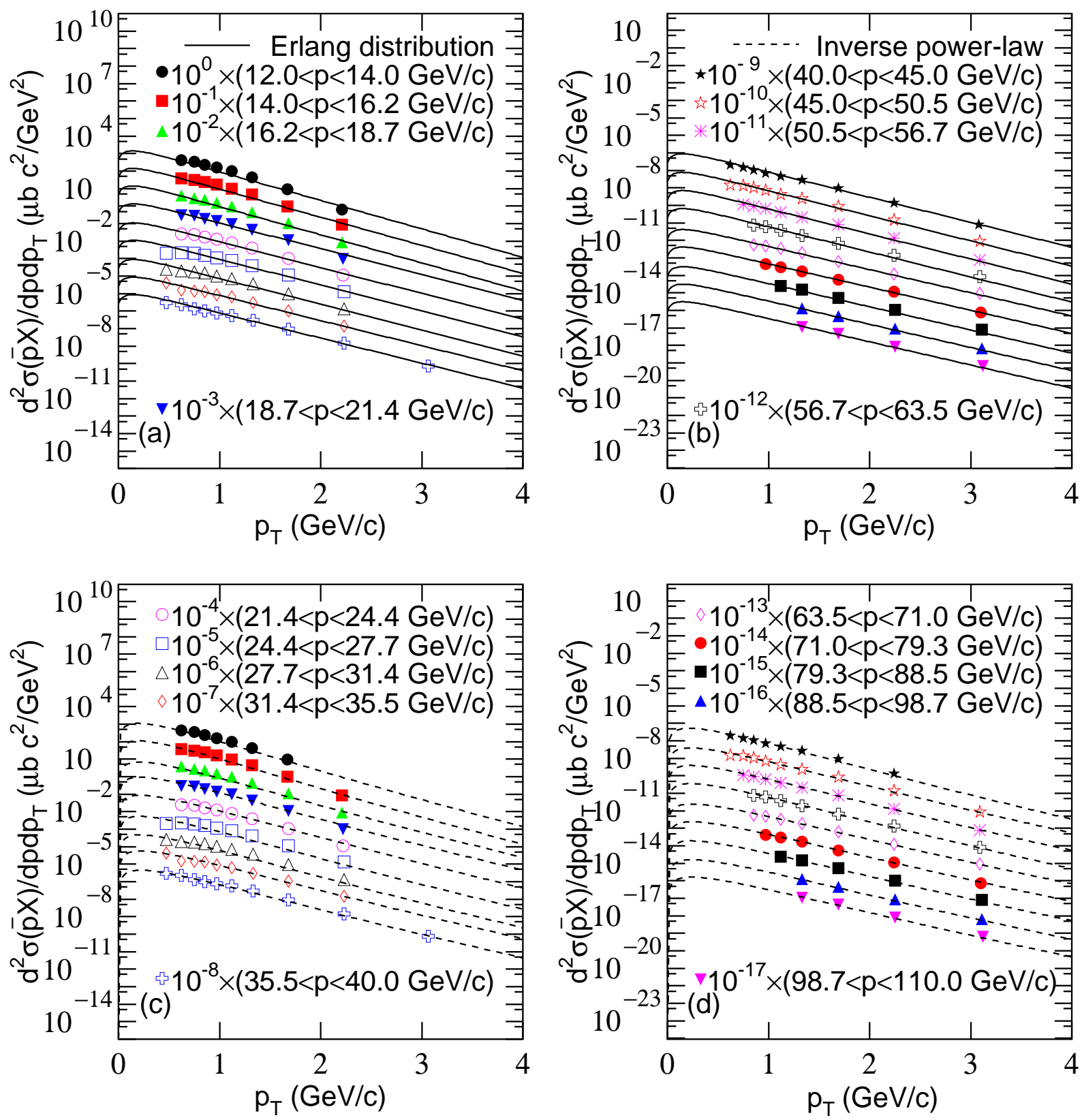

Fig. 2. Antiproton production double differential cross-section as a function of transverse momentum, integrated over various momentum ranges and divided by the ranges, in $p+\mathrm{He}$ collisions at $110 \mathrm{GeV}$. The data represent the results of LHCb Collaboration [40]. The solid and dashed curves represent the results fitted by the Erlang distribution Eq. (2) and the inverse power-law Eq. (3), where the Monte Carlo calculation is used to transform transverse momenta to momenta.

to obtain the parameter values. The values of $\left\langle p_{T}\right\rangle, p_{0}$, and $n$ are listed in Table 1 with $\chi^{2}$ per degree of freedom (dof). One can see that the LHCb experimental data on antiproton momenta in given transverse momentum ranges in $p+\mathrm{He}$ collisions at $\sqrt{s_{N N}}=110 \mathrm{GeV}$ are approximately fitted by the Erlang distribution and the inverse power-law, where the Monte Carlo calculation is used to transform transverse momenta to momenta so that the momentum distributions can be obtained.

Figure 2 shows the transverse momentum spectra, $d^{2} \sigma(\bar{p} X) / d p d p_{T}$, of $\bar{p}$ produced in $p+$ He collisions at $110 \mathrm{GeV}$, where $X$ denotes other products except for $\bar{p}$. The symbols represent the LHCb experimental data in different momentum ranges [40], which are scaled by different amounts marked in the panels (a)-(d). The data points are fitted by the Erlang distribution Eq. (2) 

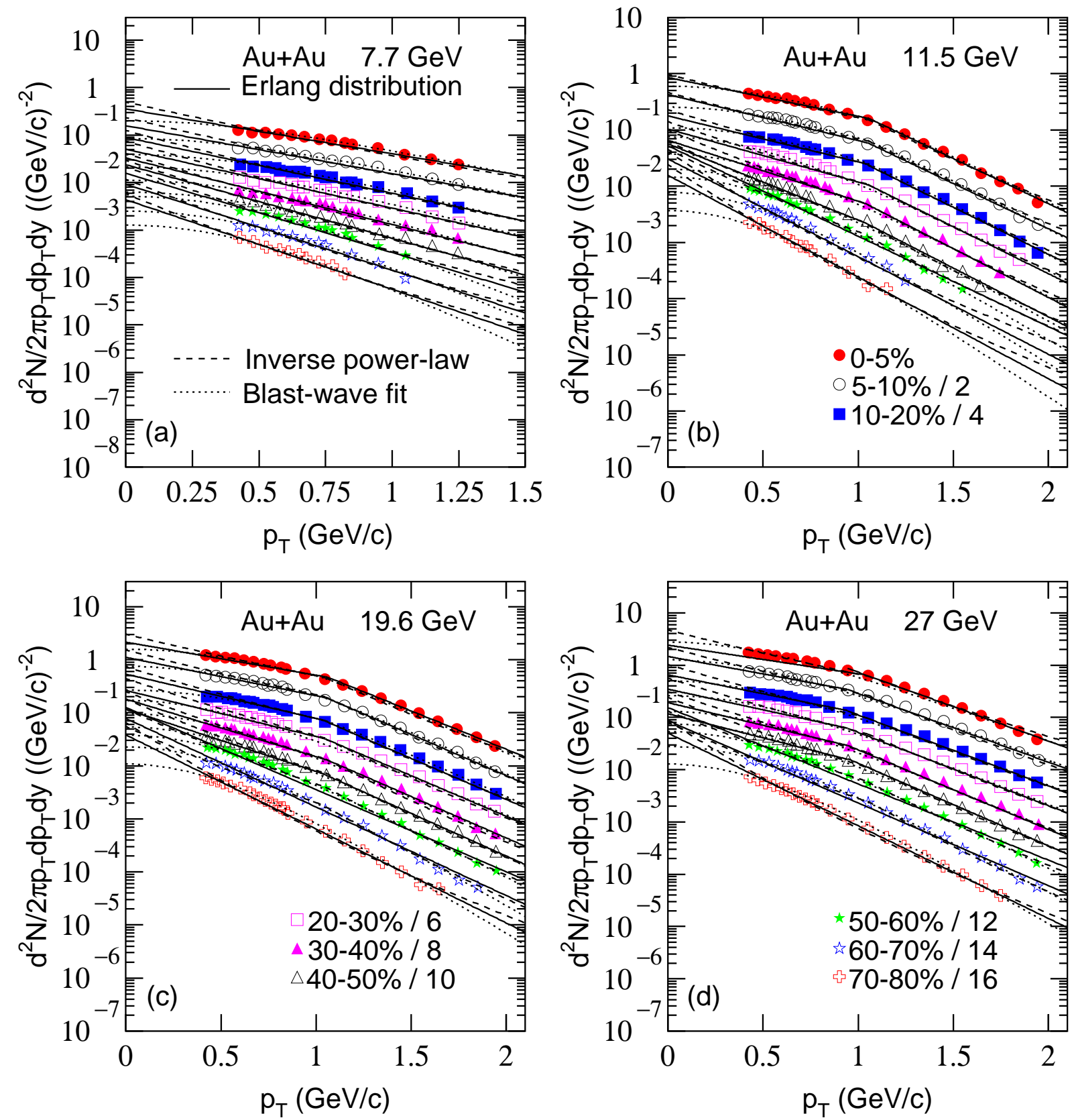

Fig. 3. Transverse momentum spectra, $d^{2} N / 2 \pi p_{T} d p_{T} d y$, of $\bar{p}$ produced in mid-rapidity $|y|<0.5$ in $\mathrm{Au}+\mathrm{Au}$ collisions at four energies: (a) 7.7, (b) 11.5 , (c) 19.6 and (d) $27 \mathrm{GeV}$. The symbols represent the experimental data measured by the STAR Collaboration in different centrality intervals [41], which are scaled by different amounts marked in the panels. The solid, dashed, and dotted curves are our results fitted by using the Erlang distribution Eq. (2), the inverse power-law Eq. (3), and the blast-wave fit Eq. (5), respectively.

(the solid curves) and the inverse power-law Eq. (3) (the dashed curves) respectively. In the fit, the Monte Carlo calculation is used to select the momentum ranges and the method of least square is used to obtain the parameter values. The values of $\left\langle p_{T}\right\rangle, p_{0}$, and $n$ are listed in Table 1 with $\chi^{2} /$ dof. One can see that the LHCb experi- mental data on antiproton transverse momenta in given momentum ranges in $p+\mathrm{He}$ collisions at $110 \mathrm{GeV}$ are approximately fitted by the Erlang distribution and the inverse power-law, where the Monte Carlo calculation is used to transform transverse momenta to momenta so that the momentum ranges can be determined. 

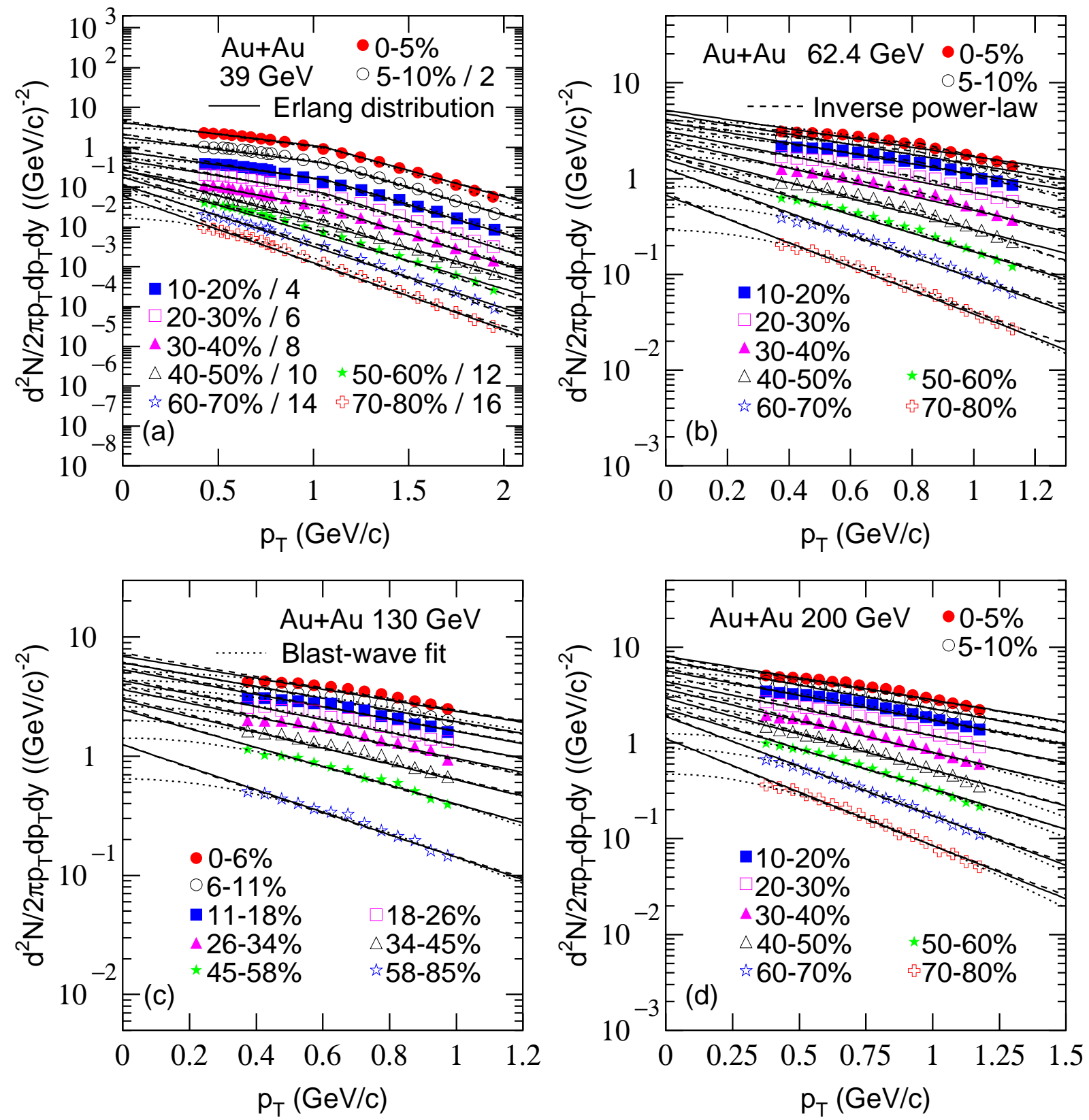

Fig. 4. Same as Fig. 3, but showing the results in $|y|<0.5$ at (a) $39 \mathrm{GeV}$ and in $|y|<1$ at (b) $62.4 \mathrm{GeV}$, (c) $130 \mathrm{GeV}$, and (d) $200 \mathrm{GeV}$, with respective centrality classes. The data are taken from refs. [14, 41].

The transverse momentum spectra, $d^{2} N / 2 \pi p_{T} d p_{T} d y$, of $\bar{p}$ produced in mid-rapidity interval $(|y|<0.5)$ in gold-gold $(\mathrm{Au}+\mathrm{Au})$ collisions at $\sqrt{s_{N N}}=$ (a) $7.7 \mathrm{GeV}$, (b) $11.5 \mathrm{GeV}$, (c) $19.6 \mathrm{GeV}$, and (d) $27 \mathrm{GeV}$ are presented in Fig. 3. Different symbols represent the data measured by the STAR Collaboration in the collision centrality classes of $0-5 \%, 5-10 \%, 10-20 \%, 20-30 \%$, $30-40 \%, 40-50 \%, 50-60 \%, 60-70 \%$, and $70-80 \%$ [41] and scaled by different amounts marked in the panels. The solid, dashed, and dotted curves are our results fitted by using the Erlang distribution Eq. (2), the inverse power-law Eq. (3), and the blast-wave fit Eq. (5), respectively. The method of least square is used to obtain the parameter values which are listed in Table 2, where only $T_{i}$ obtained from Eq (2) in the range of data available are listed. In some cases, a two-component superposed by usual step function is used [42]. The parameter values listed in Table 2 are then averaged by weighting the two components. Figure 4 is the same as Fig. 3, but showing the results in $\mathrm{Au}+\mathrm{Au}$ collisions at 

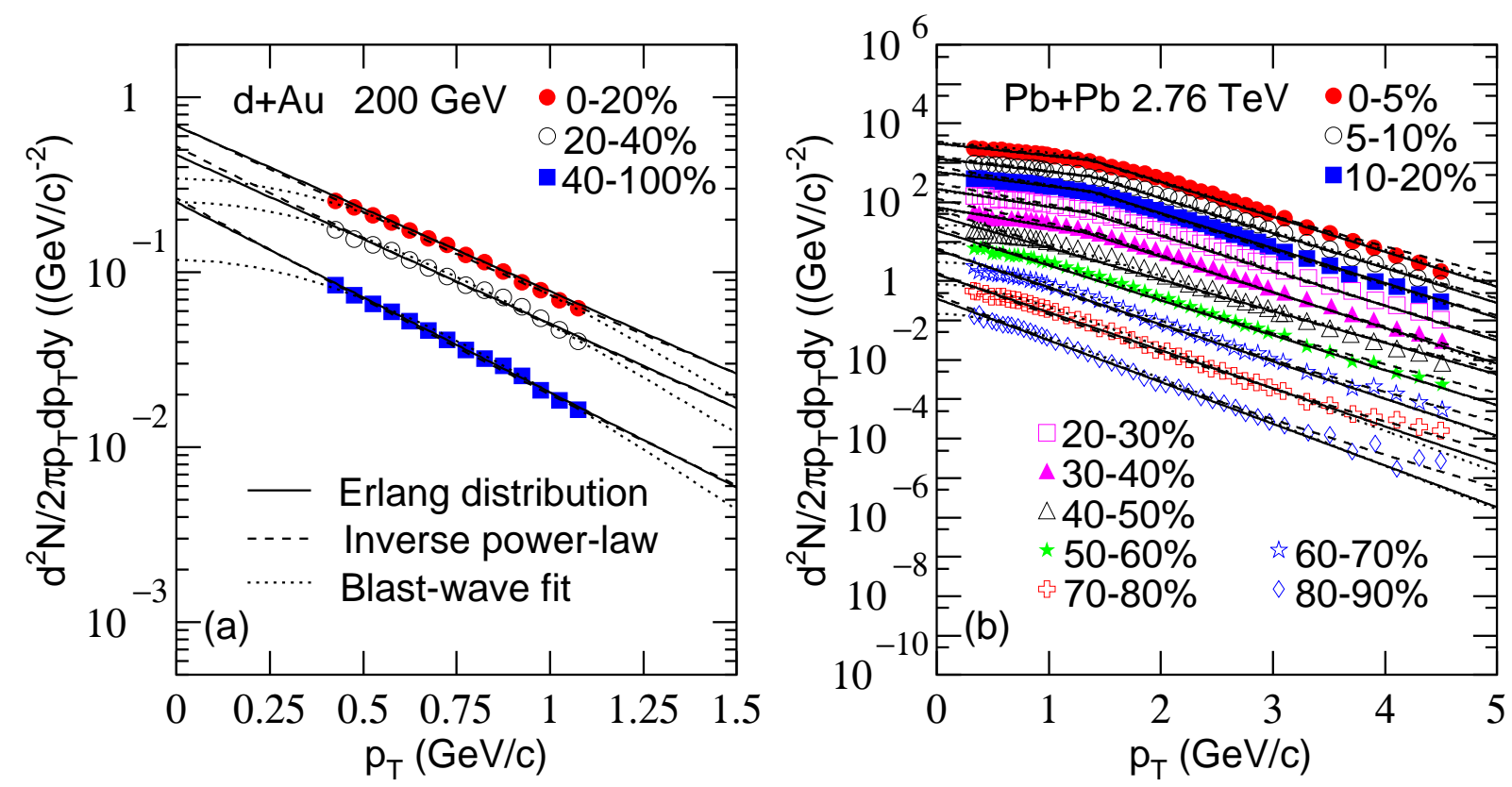

Fig. 5. Same as Fig. 3, but showing the results in $|y|<1$ in (a) $d+\mathrm{Au}$ collisions at $200 \mathrm{GeV}$ and (b) $\mathrm{Pb}+\mathrm{Pb}$ collisions at $2.76 \mathrm{TeV}$, with respective centrality classes. The data are taken from refs. [14, 43].

$\sqrt{s_{N N}}=$ (a) $39 \mathrm{GeV}$ with $|y|<0.5$ and (b) $62.4 \mathrm{GeV}$, (c) $130 \mathrm{GeV}$, and (d) $200 \mathrm{GeV}$ with $|y|<1$, with respective centrality class shown in the panels. The data are taken from refs. [14, 41]. Figure 5 is also the same as Fig. 3, but showing the results in $|y|<1$ in (a) deutongold $(d+\mathrm{Au})$ collisions at $200 \mathrm{GeV}$ and (b) lead-lead $(\mathrm{Pb}+\mathrm{Pb})$ collisions at $2.76 \mathrm{TeV}$ with respective centrality class. The data are taken from refs. [14, 43]. One can see that the STAR and LHCb experimental data on antiproton transverse momenta in different centrality classes in $\mathrm{Au}+\mathrm{Au}, d+\mathrm{Au}$, and $\mathrm{Pb}+\mathrm{Pb}$ collisions at high energies are approximately fitted by the Erlang distribution, the inverse power-law, and the blast-wave fit, though in some cases the two-component is needed.

Figure 6 shows the dependences of parameters (a)(b) $\left\langle p_{T}\right\rangle$ and $(\mathrm{c})(\mathrm{d}) T_{i}$ on (a)(c) collision energy $\left(\sqrt{s_{N N}}\right)$ in different centrality classes and (b)(d) event centrality $(C)$ at different energies. The different symbols represent the parameter values extracted from Figs. 3-5 and listed in Table 2, where only the Erlang distribution in the ranges of data available is used. In the ranges of data available, other two fits present similar results to the Erlang distribution. One can see that $\left\langle p_{T}\right\rangle$ and $T_{i}$ increase slightly with the increases of collision energy and event centrality, where the centrality $0-5 \%$ is the largest in the data samples cited in this work.

Figure 7 is the same as Fig. 6, but showing the dependences of parameters (a)(b) $T_{0}$ and (c)(d) $\beta_{T}$ on (a)(c) $\sqrt{s_{N N}}$ and (b)(d) $C$. The different symbols represent the parameter values extracted from Figs. $3-5$ and listed in Table 2, where only the blast-wave fit is used. One can see that $T_{0}$ and $\beta_{T}$ increase slightly with the increases of collision energy and event centrality. The trends of $T_{0}, \beta_{T},\left\langle p_{T}\right\rangle$, and $T_{i}$ are consistent with each other. These results are natural due to the fact that the system at high energy and with central centrality stays at the state with high degrees of excitation and expansion, in which large energy is deposited.

Figure 8 is the same as Fig. 6, but showing the dependences of parameters (a)(b) $p_{0}$ and (c)(d) $n$ on (a) (c) $\sqrt{s_{N N}}$ and (b)(d) C. The different symbols represent the parameter values extracted from Figs. 3-5 and listed in Table 2, where only the inverse power-law is used. One can see that, similar to other parameters $\left(T_{0}, \beta_{T},\left\langle p_{T}\right\rangle\right.$, and $\left.T_{i}\right)$ discussed above, $p_{0}$ also increases (slightly) with the increases of collision energy and event centrality. That is, $p_{0}$ also describes the excitation and expansion degrees of emission source, which results in large $p_{0}$ at high energy and in central collisions. Although $n$ increases slightly with the increase of collision energy, it decreases slightly with the increase of event centrality. We think that $n$ also describes the contribution fraction of hard scattering process, which results in large $n$ at high energy and in peripheral collisions. 

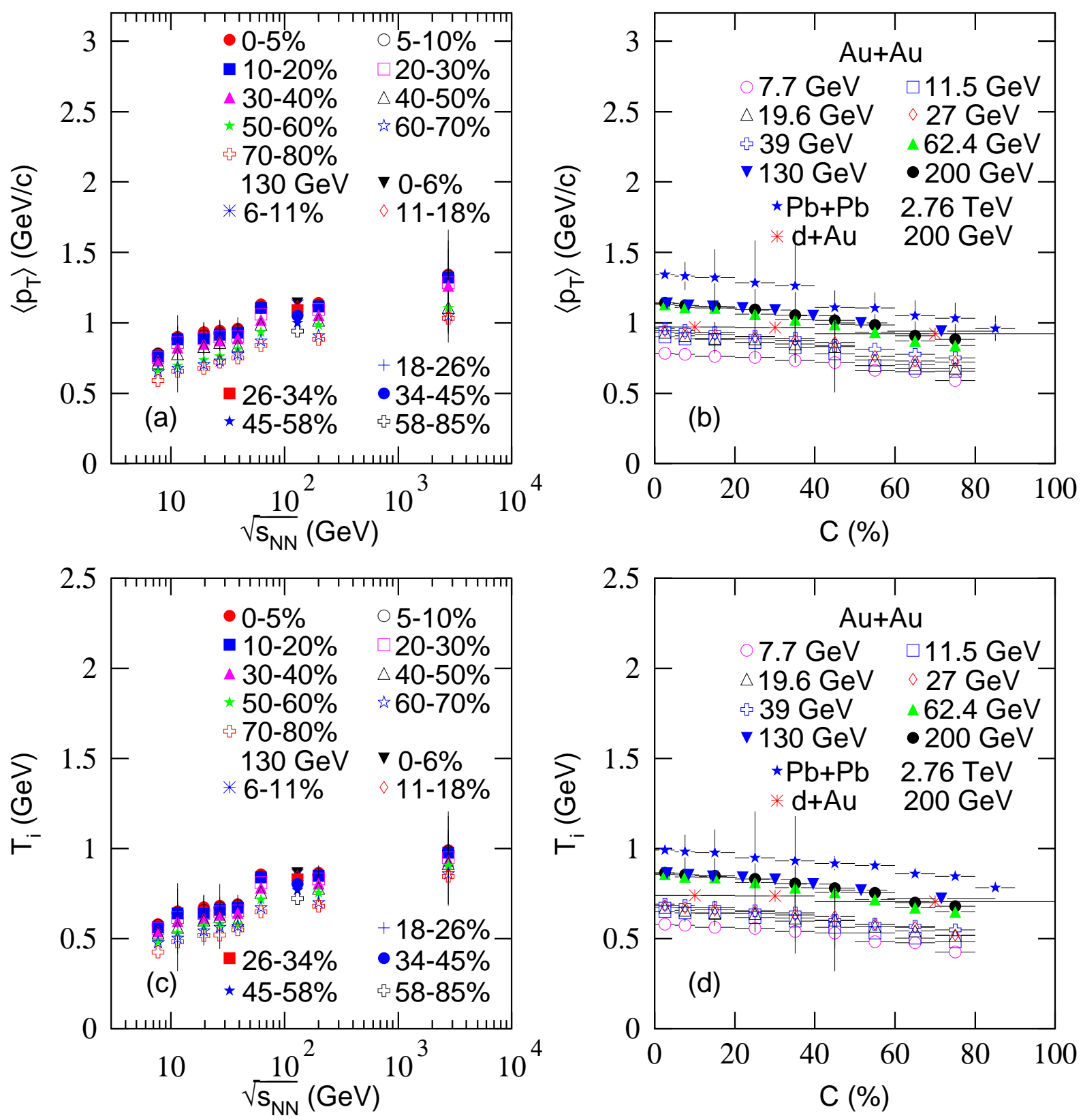

Fig. 6. Dependences of (a)(b) $\left\langle p_{T}\right\rangle$ and (c)(d) $T_{i}$ on (a)(c) $\sqrt{s_{N N}}$ and (b)(d) $C$. The different symbols represent the parameter values extracted from Figs. $3-5$ and listed in Table 2, where only the Erlang distribution in the ranges of data available is used.

There are fluctuations in the excitation functions (energy dependences) of considered parameters. These fluctuations can be regarded as the statistical fluctuations. To smooth these statistical fluctuations, more analyses are needed in future. In particular, more analyses are needed at energies below a few $\mathrm{GeV}$ which is even below the energy range of beam energy scan program [41]. We are very interested in this energy range due to the fact that it possibly contains the critical en- ergy of phase transformation from hadronic matter to quark-gluon plasma. The excitation functions of some parameters are expected to appear with the minimum, maximum, corner, saturation, and/or limitation. The starting points of saturation and limitation are particularly worth to take attention.

Before conclusions, we would like to point out the mass dependence of main parameters [34, 42, 44, 45]. Generally, $\left\langle p_{T}\right\rangle, T_{i}$, and $T_{0}$ increase with the increase of 

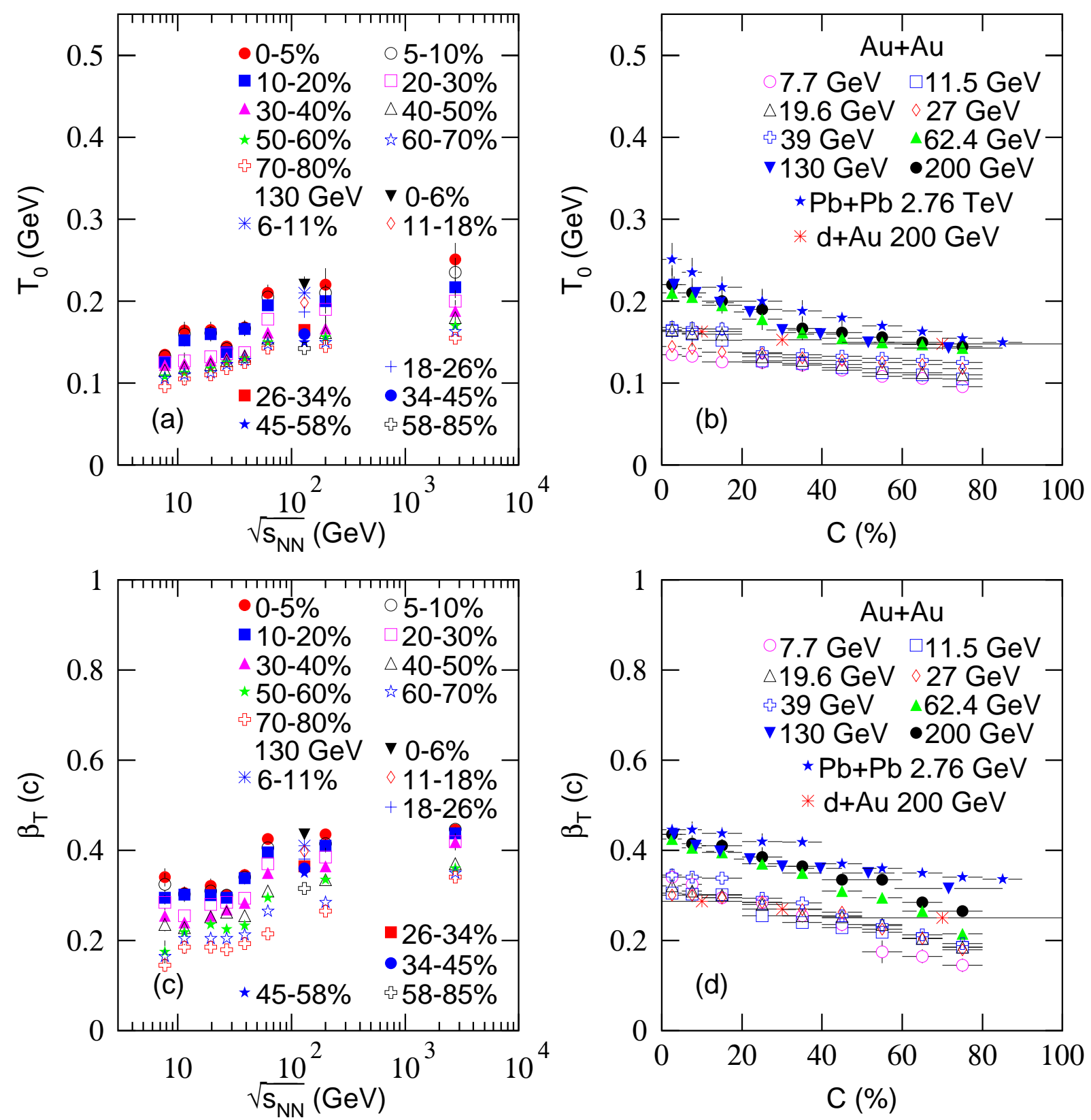

Fig. 7. Same as Fig. 6, but showing the dependences of (a)(b) $T_{0}$ and (c)(d) $\beta_{T}$ on (a)(c) $\sqrt{s_{N N}}$ and (b)(d) $C$. Only the blast-wave fit is used.

particle mass due to heavier particle corresponding to larger energy deposition. Contrarily, $\beta_{T}$ decreases with the increase of particle mass due to heavier particle having larger inertia. Although the absolute values of some parameters are model-dependent, the relative sizes are considerable. The average parameter can be obtained by weighting different yields of various particles. The weighted average of parameter values for various particles can be regarded as the mass-independent parameter value for given collisions. If the mass-independent pa- rameter means simultaneous production and freeze-out, the mass-dependent parameter implies non-single scenario $[46,47]$.

It is well known that nowadays the Tsallis distribution [48-50] is quite of use and seems to be very successful. In our previous work $[4,34,42,44,45,51,52]$, we have used the Tsallis distribution and related functions to analyze the particle production in high energy collisions. To express the variousness of fit functions, we have used other functions in this work. It is shown that 

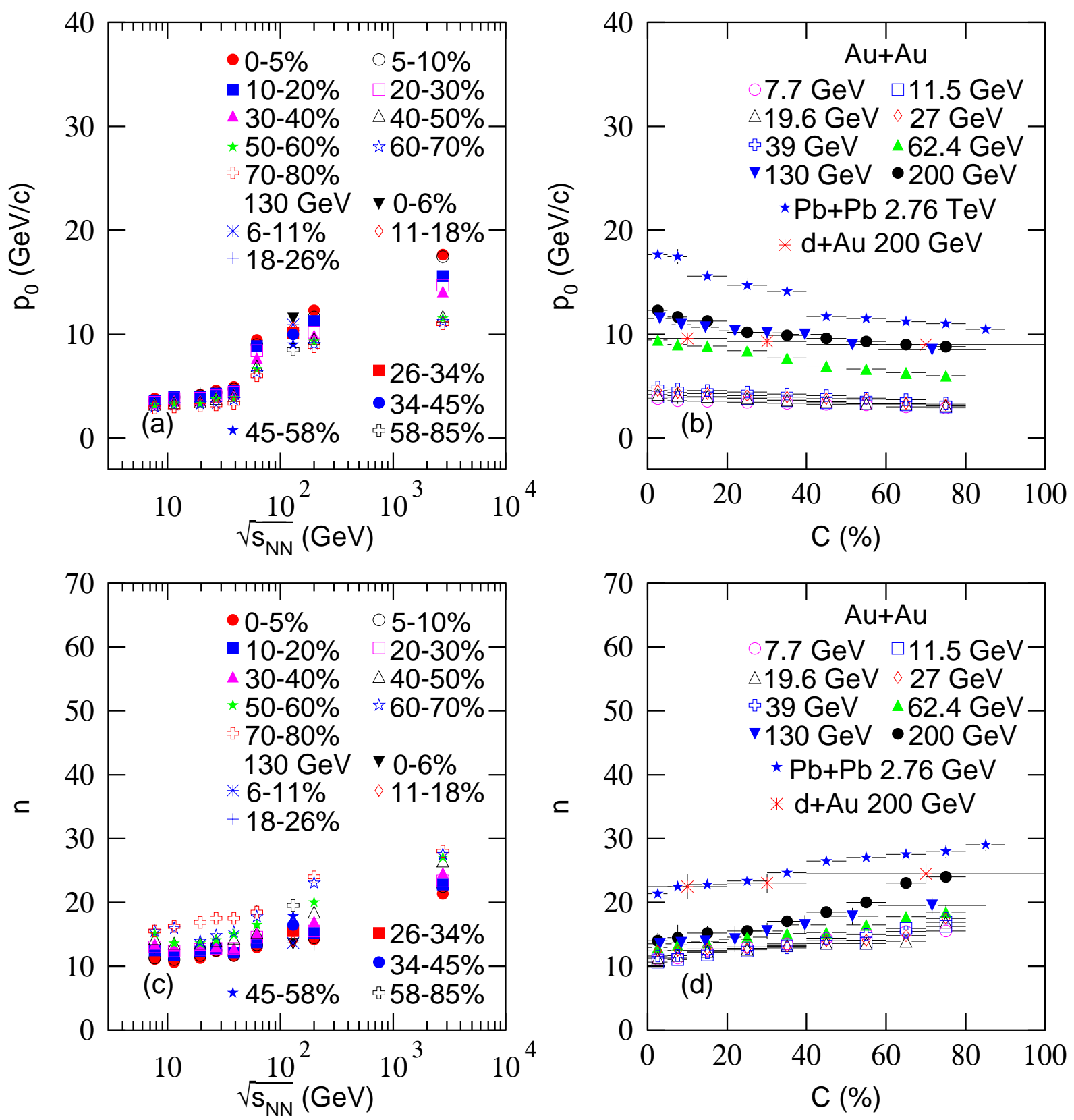

Fig. 8. Dependences of parameters (a,b) $T_{0},(\mathrm{c}, \mathrm{d}) \beta_{T}$ on energy in different centrality intervals (left panel) and on centrality at different energies (right panel). The different symbols represent the parameter values extracted from fig. 3, 4, 5 and listed in table 2, where the Blast-wave model is used for $\bar{p}$ spectra. The lines are our fitted results.

we may use different functions to extract some main parameters such as $\left\langle p_{T}\right\rangle, T_{i}, T_{0}$, and $\beta_{T}$. Among these parameters, $\left\langle p_{T}\right\rangle$ and $T_{i}$ are model-independent, while $T_{0}$ and $\beta_{T}$ are model-dependent. We hope to structure a model-independent method to extract $T_{0}$ and $\beta_{T}$ in the near future.

\section{Conclusions}

To conclude, the momentum or transverse momentum spectra of $\bar{p}$ produced at mid-rapidity in $p+\mathrm{He}$, $\mathrm{Au}+\mathrm{Au}, d+\mathrm{Au}$, and $\mathrm{Pb}+\mathrm{Pb}$ collisions over an energy range from a few $\mathrm{GeV}$ to a few $\mathrm{TeV}$ have been analyzed by the Erlang distribution, the inverse power-law (the Hagedorn function), and the blast-wave fit. In some 
cases, the usual step function is used to superpose the two-component distribution. The model results are in agreement with the experimental data of the STAR, ALICE, and LHCb Collaborations. The values of related parameters are extracted from the fit process and the excitation functions of these parameters are obtained.

The excitation functions of parameters $\left\langle p_{T}\right\rangle, T_{i}, T_{0}$, $\beta_{T}$, and $p_{0}$ increase (slightly) from a few $\mathrm{GeV}$ to a few $\mathrm{TeV}$ and from peripheral to central collisions. These trends render that these parameters describe the excitation and expansion degrees of the system. At high energy and in central collisions, large collision energy is deposited in the system, which results in high excitation and expansion degrees. The excitation function of parameter $n$ shows a slight increase from a few $\mathrm{GeV}$ to a few $\mathrm{TeV}$ and a slight decrease from peripheral to central collisions. These trends render that the parameter $n$ also describes the contribution fraction of hard scattering process. At high energy and in peripheral collisions, the hard scattering process happens with large probability.

\section{Data availability}

The data used to support the findings of this study are included within the article and are cited at relevant places within the text as references.

\section{Compliance with ethical standards}

The authors declare that they are in compliance with ethical standards regarding the content of this paper.

\section{Conflict of Interest}

The authors declare that they have no conflict of interest regarding the publication of this paper.

\section{Acknowledgments}

This work was supported by the National Natural Science Foundation of China under Grant Nos. 11575103 and 11847311, the Scientific and Technological Innovation Programs of Higher Education Institutions in Shanxi (STIP) under Grant No. 201802017, the Shanxi Provincial Natural Science Foundation under Grant No. 201701D121005, the Fund for Shanxi "1331 Project" Key Subjects Construction

\section{References}

[1] Puglisi, A., Sarracino, A., Vulpiani, A.: Temperature in and out of equilibrium: a review of concepts, tools and attempts. Phys. Rep. 709, 1-60 (2017)
[2] Bhattacharyya, T., Cleymans, J., Khuntia A. et al.: Radial flow in non-extensive thermodynamics and study of particle spectra at LHC in the limit of small $(q-1)$. Eur. Phys. J. A52, 30 (2016)

[3] Song, H.-C., Zhou, Y., Gajdošová, K.: Collective flow and hydrodynamics in large and small systems at the LHC. Nucl. Sci. Tech. 28, 99 (2017)

[4] Wei, H.-R., Liu, F.-H., Lacey, R. A.: Kinetic freeze-out temperature and flow velocity extracted from transverse momentum spectra of final-state light flavor particles produced in collisions at RHIC and LHC. Eur. Phys. J. A52, 102 (2016)

[5] Gao, L.-N., Liu, F.-H., Li, B.-C.: Rapidity dependent transverse momentum spectra of heavy quarkonia produced in small collision systems at the LHC. Adv. High Energy Phys. 2019, 6739315 (2019)

[6] Liu, F.-H.: Particle production in Au-Au collisions at RHIC energies. Phys. Lett. B583, 68-72 (2004)

[7] Liu, F.-H., Li, J.-S.: Isotopic production cross section of fragments in ${ }^{56} \mathrm{Fe}+\mathrm{p}$ and ${ }^{136} \mathrm{Xe}\left({ }^{124} \mathrm{Xe}\right)+\mathrm{Pb}$ reactions over an energy range from $300 \mathrm{~A}$ to $1500 \mathrm{~A} \mathrm{MeV}$. Phys. Rev. C78, 044602 (2008)

[8] Liu, F.-H.: Unified description of multiplicity distributions of final-state particles produced in collisions at high energies. Nucl. Phys. A810, 159-172 (2008)

[9] Liu, F.-H., Gao, Y.-Q., Tian, T., Li, B.-C.: Unified description of transverse momentum spectrums contributed by soft and hard processes in high-energy nuclear collisions. Eur. Phys. J. A50, 94 (2014)

[10] Hagedorn, R.: Multiplicities, $p_{T}$ distributions and the expected hadron $\longrightarrow$ quark-gluon phase transition. Riv. Nuovo Cimento 6(10), 1-50 (1983).

[11] Abelev, B. et al. (ALICE Collaboration): Production of $\Sigma(1385)^{ \pm}$and $\Sigma(1530)^{0}$ in proton-proton collisions at $\sqrt{s}=7$ TeV. Eur. Phys. J. C75, 1 (2015)

[12] Schnedermann, E., Sollfrank, J., Heinz, U.: Thermal phenomenology of hadrons from $200 \mathrm{~A} \mathrm{GeV} \mathrm{S+S} \mathrm{colli-}$ sions. Phys. Rev. C48, 2462-2475 (1993)

[13] Abelev, B. I. et al. (STAR Collaboration): Systematic measurements of identified particle spectra in $\mathrm{pp}$, $\mathrm{d}+\mathrm{Au}$, and $\mathrm{Au}+\mathrm{Au}$ collisions at the STAR detector. Phys. Rev. C79, 034909 (2009)

[14] Abelev, B. I. et al. (STAR Collaboration) Identified particle production, azimuthal anisotropy, and interferometry measurements in $\mathrm{Au}+\mathrm{Au}$ collisions at $\sqrt{s_{N N}}=9.2$ GeV. Phys. Rev. C81, 024911 (2010)

[15] Tang, Z.-B., Xu, Y.-C., Ruan, L.-J., van Buren, G., Wang, F.-Q., Xu, Z.-B.: Spectra and radial flow in relativistic heavy ion collisions with Tsallis statistics in a Blast-wave description. Phys. Rev. C79, 051901(R) (2009)

[16] Kittel, W., De Wolf, E. A.: Soft Multihadron Dynamics. p. 652, World Scientific, Singapore (2005) 
[17] Sarkisyan, E. K. G., Sakharov, A. S.: On similarities of bulk observables in nuclear and particle collisions. CERN-PH-TH-2004-213 (2004), arXiv:hep-ph/0410324 (2004)

[18] Sarkisyan, E. K. G., Sakharov, A. S. Multihadron production features in different reactions. AIP Conf. Proc. 828, 35-41 (2006)

[19] Sarkisyan, E. K. G., Sakharov, A. S.: Relating multihadron production in hadronic and nuclear collisions. Eur. Phys. J. C70, 533-541 (2010)

[20] Nouicer, R.: Similarity of initial states in A+A and p+p collisions in constituent quarks framework. AIP Conf. Proc. 828, 11-16 (2006)

[21] Nouicer, R. for the PHOBOS Collaboration: Systematic of global observables in $\mathrm{Cu}+\mathrm{Cu}$ and $\mathrm{Au}+\mathrm{Au}$ collisions at RHIC energies. AIP Conf. Proc. 842, 86-88 (2006)

[22] Nouicer, R.: Charged particles multiplicities in A+A and $\mathrm{p}+\mathrm{p}$ collisions in the constituent quarks framework. Eur. Phys. J. C49, 281-286 (2007)

[23] Grosse-Oetringhaus, J. F., Reygers, K.: Charged particle multiplicity in proton-proton collisions. J. Phys. G37, 083001 (2010)

[24] Sarkisyan, E. K. G., Mishra, A. N., Sahoo, R., Sakharov, A. S.: Multihadron production dynamics exploring the energy balance in hadronic and nuclear collisions. Phys. Rev. D93, 054046 (2016)

[25] Sarkisyan, E. K. G., Mishra, A. N., Sahoo, R., Sakharov, A. S.: Centrality dependence of midrapidity density from $\mathrm{GeV}$ to $\mathrm{TeV}$ heavy-ion collisions in the effective-energy universality picture of hadroproduction. Phys. Rev. D94, 011501(R) (2016)

[26] Mishra, A. N., Ortiz, A., Paić, G.: Intriguing similarities of high- $p_{T}$ particle production between pp and A-A Collisions. Phys. Rev. C99, 034911 (2019)

[27] Aamodt, K. et al. (ALICE Collaboration) Transverse momentum spectra of charged particles in protonproton collisions at $\sqrt{s}=900 \mathrm{GeV}$ with ALICE at the LHC. Phys. Lett. B693, 53-68 (2010)

[28] De Falco, A. for the ALICE Collaboration: Vector meson production in pp collisions at $\sqrt{s}=7 \mathrm{TeV}$, measured with the ALICE detector. J. Phys. G38, 124083 (2011)

[29] Abelev, B. et al. (ALICE Collaboration) Light vector meson production in pp collisions at $\sqrt{s}=7 \mathrm{TeV}$. Phys. Lett. B710, 557-568 (2012)

[30] Adare, A. et al. (PHENIX Collaboration): Nuclear modification factors of $\phi$ mesons in $\mathrm{d}+\mathrm{Au}, \mathrm{Cu}+\mathrm{Cu}$, and $\mathrm{Au}+\mathrm{Au}$ collisions at $\sqrt{s_{N N}}=200 \mathrm{GeV}$. Phys. Rev. C83, 83, 024909 (2011)

[31] Abelev, B. et al. (ALICE Collaboration): Inclusive $J / \psi$ production in pp collisions at $\sqrt{s}=2.76 \mathrm{TeV}$. Phys. Lett. B718, 295-306 (2012)
[32] Lakomov, I. for the ALICE collaboration: Event activity dependence of inclusive $J / \psi$ production in p- $\mathrm{Pb}$ collisions at $\sqrt{s_{N N}}=5.02 \mathrm{TeV}$ with ALICE at the LHC. Nucl. Phys. A931, 1179-1183 (2014)

[33] Abelev, B. et al. (ALICE Collaboration): Heavy flavour decay muon production at forward rapidity in protonproton collisions at $\sqrt{s}=7 \mathrm{TeV}$. Phys. Lett. B708, 265-275 (2012)

[34] Lao, H.-L., Liu, F.-H., Li, B.-C., Duan, M.-Y.: Kinetic freeze-out temperatures in central and peripheral collisions: which one is larger?. Nucl. Sci. Tech. 29, 82 (2018)

[35] Robert, C. P., Casella, G.: Monte Carlo Statistical Methods: Second Edition, Springer, Germany and World Scientific, Sigapore, (2009)

[36] Gutay, L. G., Hirsch A. S., Pajares, C., Scharenberg R. P. and Srivastava B. K.: De-confinement in small systems: clustering of color sources in high multiplicity $\bar{p}$ p collisions at $\sqrt{s_{N N}}=1.8 \mathrm{TeV}$. Int. J. Mod. Phys. E24, 1550101 (2015)

[37] Hirsch A. S., Pajares C., Scharenberg R. P. and Srivastava B. K.: De-confinement in high multiplicity protonproton collisions at LHC energies. arXiv:1803.02301 [hep-ph] (2018)

[38] Sahoo P., De S., Tiwari S. K. and Sahoo R.: Energy and centrality dependent study of deconfinement phase transition in a color string percolation approach at RHIC energies. Eur. Phys. J. A54, 136 (2018)

[39] Aaij, R. et al. (LHCb collaboration) Measurement of antiproton production in pHe collisions at $\sqrt{s_{N N}}=110$ GeV. Phys. Rev. Lett. 121, 222001 (2018)

[40] Graziani, G. for the LHCb Collaboration: Measurement of antiproton production in pHe collisions at $\sqrt{s_{N N}}=$ $110 \mathrm{GeV}$. Conference report prepared for the 52nd Rencontres de Moriond on Electroweak Interactions and Unified Theories, 18-25 March 2017, La Thuile, Italy, CERN-LHCb-CONF-2017-002 (2017)

[41] Adamczyk, L. et al. (STAR Collaboration) Bulk properties of the medium produced in relativistic heavy-ion collisions from the beam energy scan program. Phys. Rev. C96, 044904 (2017)

[42] Lao, H.-L., Liu, F.-H., Li, B.-C., Duan, M.-Y., Lacey, R. A.: Examining the model dependence of the determination of kinetic freeze-out temperature and transverse flow velocity in small collision system. Nucl. Sci. Tech. 29, 164 (2018)

[43] Abelev, B. et al. (ALICE Collaboration) Centrality dependence of $\pi, K$, and $p$ production in $\mathrm{Pb}-\mathrm{Pb}$ collisions at $\sqrt{s_{N N}}=2.76 \mathrm{TeV}$. Phys. Rev. C88, 044910 (2013)

[44] Li, L.-L., Liu, F.-H.: Excitation functions of kinetic freeze-out temperature and transverse flow velocity in proton-proton collisions. arXiv:1805.03342 [hep-ph] (2018) 
[45] Li, L.-L., Liu, F.-H.: Energy dependent kinetic freezeout temperature and transverse flow velocity in high energy collisions. Eur. Phys. J. A54, 169 (2018)

[46] Waqas, M., Liu, F.-H., Fakhraddin, S., Rahim, M. A.: Possible scenarios for single, double, or multiple kinetic freeze-out in high-energy collisions. Indian J. Phys. 93, https://doi.org/10.1007/s12648-019-01396-9 (2019)

[47] Lao, H.-L., Wei, H.-R., Liu, F.-H., Lacey, R. A.: An evidence of mass-dependent differential kinetic freeze-out scenario observed in $\mathrm{Pb}-\mathrm{Pb}$ collisions at 2.76 $\mathrm{TeV}$. Eur. Phys. J. A52, 203 (2016)

[48] Tsallis, C.: Possible generalization of Boltzmann-Gibbs statistics. J. Stat. Phys. 52, 479-487 (1988)

[49] Cleymans, J., Worku, D.: Relativistic thermodynam- ics: transverse momentum distributions in high-energy physics. Eur. Phys. J. A48, 160 (2012)

[50] Zheng, H., Zhu, L. L.: Comparing the Tsallis distribution with and without thermodynamical description in p + p collisions. Adv. High Energy Phys. 2016, 9632126 (2016)

[51] Gao, L.-N., Liu, F.-H., Lacey, R. A.: Excitation functions of parameters in Erlang distribution, Schwinger mechanism, and Tsallis statistics in RHIC BES program. Eur. Phys. J. A52, 137 (2016)

[52] Lao, H.-L., Liu, F.-H., Lacey, R. A.: Extracting kinetic freeze-out temperature and radial flow velocity from an improved Tsallis distribution. Eur. Phys. J. A53, 44 (2017) 